\title{
Third-Generation Antiseizure Medications for Adjunctive Treatment of Focal-Onset Seizures in Adults: A Systematic Review and Network Meta-analysis
}

\author{
Simona Lattanzi ${ }^{1}$ (D) Eugen Trinka ${ }^{2,3,4} \cdot$ Gaetano Zaccara $^{5} \cdot$ Pasquale Striano $^{6} \cdot$ Emilio Russo $^{7} \cdot$ Cinzia Del Giovane $^{8}$. \\ Mauro Silvestrini ${ }^{1} \cdot$ Francesco Brigo $^{9,10}$
}

Accepted: 30 November 2021 / Published online: 21 January 2022

(c) The Author(s) 2022

\begin{abstract}
Background Brivaracetam (BRV), cenobamate (CNB), eslicarbazepine acetate (ESL), lacosamide (LCM) and perampanel (PER) are antiseizure medications (ASMs) approved for adjunctive treatment of focal-onset seizures. So far, no randomised controlled trial directly compared the efficacy and safety of these drugs.

Objective We estimated the comparative efficacy and safety of these ASMs for the treatment of focal-onset seizures in adults with epilepsy using a network meta-analysis (NMA).

Methods We systematically searched (June week 4, 2021) MEDLINE (accessed by PubMed), the Cochrane Central Register of Controlled Trials (CENTRAL), and the US National Institutes of Health Clinical Trials Registry (http://www.clinicaltr ials.gov). There were no date limitations or language restrictions. Randomised, double-blinded, controlled, parallel-group, add-on studies that compared oral BRV, CNB, ESL, LCM, and PER versus any comparator over maintenance periods of at least 12 weeks and included adult patients with focal seizures uncontrolled by concomitant ASMs were identified. The efficacy outcomes were the proportions of patients with $\geq 50 \%$ and $100 \%$ reduction in baseline seizure frequency during the maintenance period. The tolerability outcomes were the proportions of participants who experienced at least one treatmentemergent adverse event (TEAE) and experienced at least one TEAE leading to discontinuation. Effect sizes were estimated by network meta-analyses within a frequentist framework. The hierarchy of competing interventions was established using the surface under the cumulative ranking curve (SUCRA).

Results Sixteen trials (BRV: $n=3$, CNB: $n=1$, ESL: $n=4$, LCM: $n=4$, PER: $n=4$ ) were included, overall enrolling 4507 patients randomised to add-on active treatments $(\mathrm{BRV}=803, \mathrm{CNB}=221, \mathrm{ESL}=990, \mathrm{LCM}=1104$, and $\mathrm{PER}=1389)$ and 2246 to add-on placebo. Cenobamate was associated with a higher rate of $\geq 50 \%$ seizure frequency reduction than BRV [odds ratio (OR) 2.02, 95\% confidence interval (CI) 1.11-3.66], ESL (OR 1.93, 95\% CI 1.07-3.48), LCM (OR 1.86, 95\% CI 1.04-3.32), and PER (OR 2.07, 95\% CI 1.16-3.70). There was a not statistically significant trend favouring CNB over ESL, LCM and PER for the seizure freedom outcome. Brivaracetam (OR 0.61, 95\% CI 0.44-0.86) and LCM (OR 0.60, 95\% CI 0.40-0.88) were associated with a lower proportion of participants experiencing TEAEs compared to ESL, and patients treated with PER were associated with a higher risk to experience at least one TEAE (OR 1.42, 95\% CI 1.02-1.96) than BRV. According to SUCRA, CNB had the greatest likelihood of being the best option for the $\geq 50 \%$ and $100 \%$ seizure frequency reduction, and BRV and LCM had the highest probabilities of being the best-tolerated treatments.

Conclusions Cenobamate ranked best for efficacy, and BRV and LCM were best tolerated over the other comparators. Although NMAs cannot replace direct comparisons, they may support physicians in clinical decision making.

\section{Introduction}

Epilepsies are among the most common chronic disorders of the brain and affect approximately 70 million people

Simona Lattanzi

alfierelattanzisimona@gmail.com

Extended author information available on the last page of the article

worldwide [1-3]. The management of epilepsy is mainly symptomatic, aimed at reducing the risk of seizure recurrence, and antiseizure medications (ASMs) are the mainstay of treatment. However, more than $50 \%$ of patients do not achieve seizure freedom with initial monotherapy [4-7], and treatment options for these patients include other ASM monotherapies or combination therapy using additional ASMs as adjunctive therapy. Focal onset seizures are the most
\end{abstract}




\section{Key Points}

Brivaracetam, cenobamate, eslicarbazepine acetate, lacosamide, and perampanel are antiseizure medications approved for adjunctive treatment of focal-onset seizures.

Adjunctive cenobamate had the greatest likelihood of being the best option for the $\geq 50 \%$ and $100 \%$ seizure frequency reduction.

Adjunctive brivaracetam and lacosamide had the highest probabilities of being the best-tolerated treatments.

prevalent type of seizures and can pose a significant risk to individual health and impair quality of life, particularly when they are associated with impairment of awareness.

Five "third-generation" ASMs were approved for adjunctive treatment of focal onset seizures in adult patients during the past 10 years: lacosamide (LCM), eslicarbazepine acetate (ESL), perampanel (PER), brivaracetam (BRV), and cenobamate (CNB) [8]. So far, however, there has not been a single randomised controlled trial (RCT) performed to directly compare the efficacy and safety of these drugs. Here, we assessed the comparative efficacy and safety of these ASMs using a network meta-analysis (NMA).

\section{Methods}

\subsection{Search Strategy}

The results were reported according to the recommendations of the Preferred Reporting Items for Systematic Reviews and Meta-Analyses (PRISMA) extension statement for network meta-analyses [9]. We systematically searched (June, Week 4, 2021) MEDLINE (accessed by PubMed), the Cochrane Central Register of Controlled Trials (CENTRAL), and the US National Institutes of Health Clinical Trials Registry (http://www.clinicaltrials.gov). The search terms were (lacosamide OR perampanel OR brivaracetam OR eslicarbazepine OR cenobamate) AND (epilepsy OR seizure); further details of search strategies are outlined in the Appendix I of the electronic supplementary material (SI). There were no date limitations or language restrictions. The reference lists of retrieved studies were reviewed to identify additional reports of relevant trials. The protocol was not registered previously.

\subsection{Eligibility Criteria}

Randomised, double-blinded, controlled, parallel-group, add-on studies that compared oral BRV, CNB, ESL, LCM, and PER versus any comparator over maintenance periods (or periods of stable dosing for dose-escalation trials) of at least 12 weeks and included adult patients with focal epilepsy and seizures uncontrolled by one or more concomitant ASMs.

\subsection{Outcome Measures}

The efficacy outcomes were the proportion of patients with $\geq 50 \%$ (seizure response) and 100\% (seizure freedom) reduction in monthly seizure frequency during the maintenance treatment period compared with the pre-randomisation baseline; if data over the maintenance period were not reported, the treatment period was used. The modified intent-to-treat (mITT) population data were used for the efficacy analyses. The mITT analysis set consisted of all randomised patients who received at least one dose of the study drug and had any seizure frequency data collected during the double-blind phase. For the seizure freedom outcome, when data were available, we adopted the "pragmatic ITT" approach, whereby only patients who complete the study and are seizure-free can be classed as seizure-free in the numerator and the mITT is used as denominator [10].

The tolerability outcomes included the proportions of participants who experienced at least one treatment-emergent adverse event (TEAE) and experienced at least one TEAE leading to discontinuation. The safety population, which included all randomly assigned patients, was considered for the tolerability analyses. For each ASM, only licensed, maintenance daily doses for adjunctive therapy of focal onset seizures in adults according to individual summaries of product characteristics (i.e., BRV $50-200 \mathrm{mg}$, CNB 200-400 mg, ESL 800-1200 mg, LCM 200-400 mg, and PER 4-12 mg) were considered [11-15].

\subsection{Study Selection, Data Extraction, Assessment of the Risk of Bias and Confidence in the Evidence}

Two review authors independently assessed trials for inclusion and extracted the following information from included studies: main study author and date of publication, methodology and trial design (methods of randomisation, allocation concealment and blinding, duration of baseline and 
treatment periods, dose/s of ASMs tested), number and demographics of participants (age, sex, ethnicity, concomitant ASMs, seizure frequency during the baseline period), and the number of patients experiencing any outcome per randomised group. Any disagreement was resolved by discussion with a third review author. The risk of bias of the identified studies was assessed following the recommendations of the Cochrane Collaboration [16]. We judged the confidence in the evidence derived from NMA using the web application CINeMA (http://cinema.ispm.ch/) [17].

\subsection{Statistical Analysis}

First, pairwise meta-analyses were performed for all outcomes, using a fixed-effects model. Second, network metaanalyses within a frequentist framework were done assuming equal heterogeneity parameter $\tau$ across all comparisons [18]. For studies with more than one randomised treatment arm within the licensed maintenance daily doses, the arms were pooled to form a single node for the corresponding ASM. Where sufficient data were available, secondary analyses were performed according to daily dosages with each dose within the licensed maintenance daily doses of any ASM representing a separate node in the network. It is appropriate to use NMA if the assumption of transitivity (distributions of the potential effect modifiers, like study and patient-level covariates, are balanced across all pairwise comparisons) can be defended [19]. We assessed the transitivity assumption by looking at the similarities of studies in each comparison. We used the loop-specific approach to evaluate coherence locally, defined as the statistical agreement between direct and indirect evidence for a specific comparison (consistency assumption) [20]. Coherence could only be assessed for the secondary analyses by doses and not for the primary analyses as there were no closed loops in the 'primary' networks. Effect sizes were estimated as odds ratios (ORs) with their $95 \%$ confidence intervals (CIs). The hierarchy of competing interventions was established using the surface under the cumulative ranking curve (SUCRA) and mean ranks. Data analysis was performed using STATA/IC 13.1 statistical package (StataCorp LP, College Station, TX, USA).

\section{Results}

\subsection{Results of the Search and Characteristics of Included Studies}

Database and trial register searching identified a total of 1100 records; 16 RCTs trials were included in the qualitative and quantitative synthesis [21-36] (Fig. 1).
All 16 RCTs were placebo-controlled studies and assessed both the efficacy and safety of the investigational drug (BRV: $n=3$, CNB: $n=1$, ESL: $n=4$, LCM: $n=4$, PER: $n=4)$. All studies had a titration phase, apart from three BRV trials; the maintenance period was 12 weeks in eleven studies, 13 weeks in four studies and 14 weeks in one study. Details of the studies are provided in Table 1. The studies recruited 6753 patients, of which 4507 were randomised to active treatments $(\mathrm{BRV}=803, \mathrm{CNB}=221$, $\mathrm{ESL}=990, \mathrm{LCM}=1104$, and PER $=1389)$ and 2246 to placebo. Participants enrolled across the studies were similar in terms of average age, sex distribution, epilepsy duration, concomitant ASMs with most patients treated with two or more ASMs, and baseline seizure frequency. Characteristics of the participants are summarised in Table 2; details of the type of concomitant ASMs are provided in Table e- 1 in the SI.

Fifteen trials were considered to have used adequate methods of random sequence generation and allocation concealment; conversely, we judged one study to have an unclear risk of selection bias as study authors failed to provide details regarding methods used for allocation concealment [29]. We judged all included trials at low risk of performance and detection bias since blinding was ensured by matching placebo, and patients, investigators, and study personnel were all masked to the treatment assignment. Regarding the risk of attrition bias, patients lost to follow-up and withdrawals with corresponding reasons were documented in all trials. Studies presenting differences between intervention groups in the proportions of imputed outcome data for the responder rate outcome were judged to have an unclear risk of bias [21, 23, 25, 28-36]. There was no suspicion of selective outcome reporting across the trials. A summary of bias assessment risk is reported in Table e- 2 in the SI.

\subsection{Efficacy Outcomes}

All RCTs provided data on the proportion of patients with $\geq 50 \%$ and $100 \%$ reduction in monthly seizure frequency from baseline. Most studies reported efficacy outcomes over the maintenance period, apart from one ESL study [27], for which treatment period was considered. In the CNB study [24], the mITT population for the responder rate outcome included patients who completed the 6-week titration phase and took at least one dose of study drug in the maintenance phase and had maintenance phase seizure data (mITT maintenance phase population). In one BRV study [22], the ITT population, defined as all randomised subjects who received at least one dose of study medication, was available for the responder rate outcome. The "pragmatic ITT" data for the seizure freedom outcome were available in most trials; in three studies, outcome up to treatment discontinuation was used to impute the seizure-freedom status for the remainder 
Fig. 1 Study flow diagram. CENTRAL Cochrane Central Register of Controlled Trials

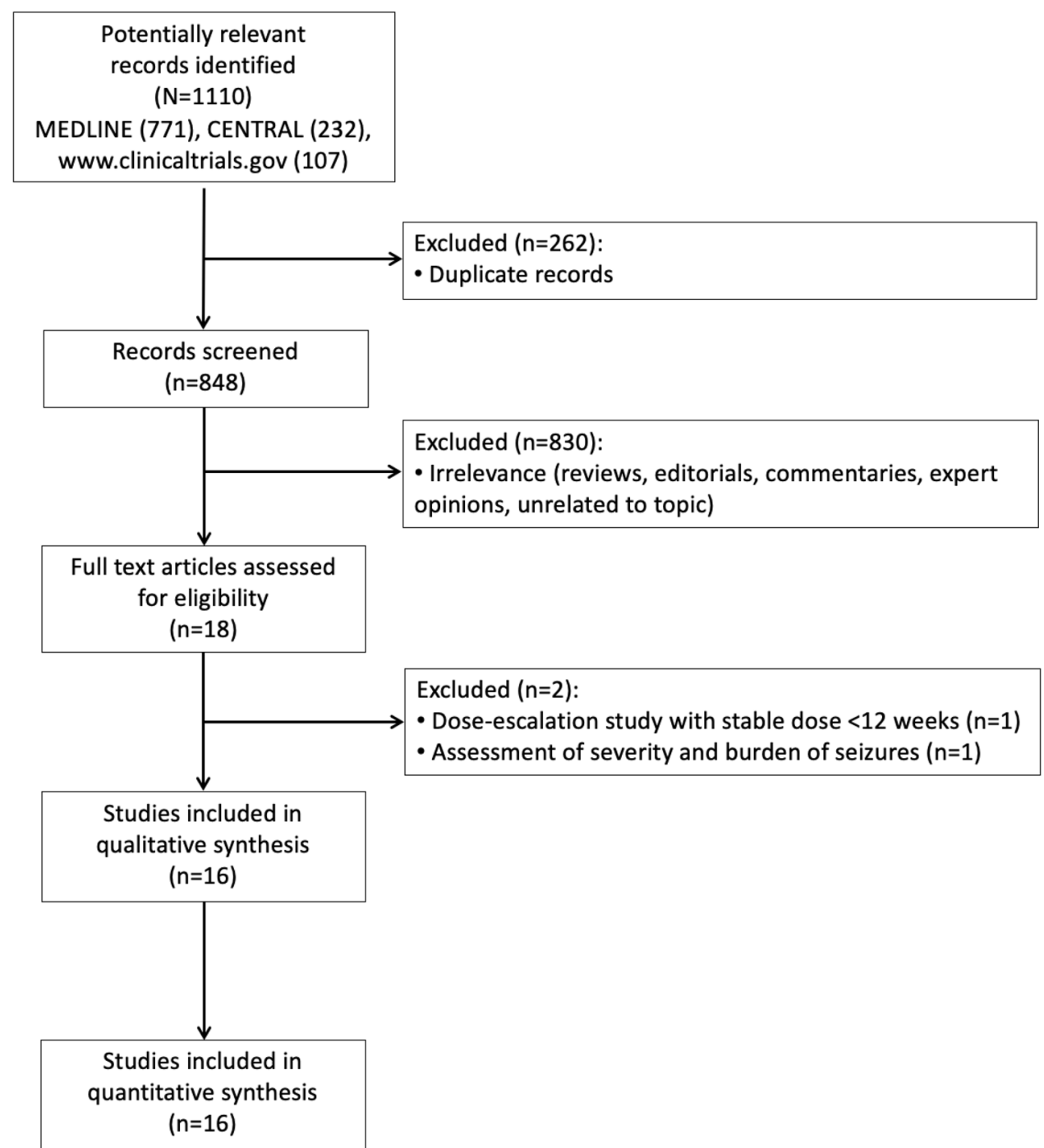

of the trial $[25,28,36]$. The network plots of treatment comparisons for the efficacy outcomes are shown in Figs. e-1 and $\mathrm{e}-2$ in the SI.

At the pairwise meta-analyses, all ASMs were associated with a higher responder rate than placebo, and BRV, CNB, ESL, and PER were associated with a higher rate of seizure freedom than placebo (Table e-3 in the SI). In the analysis by dosages, ESL at $1200 \mathrm{mg} /$ day was associated with a higher responder rate than ESL at $800 \mathrm{mg} /$ day; LCM at $400 \mathrm{mg} /$ day was associated with a higher responder rate than LCM at $200 \mathrm{mg} / \mathrm{day}$, and PER at 8 and $12 \mathrm{mg} /$ day was associated with a higher responder rate than PER at $4 \mathrm{mg} /$ day (Table $\mathrm{e}-4$ in the SI).

Results of the network meta-analyses of efficacy outcomes are shown in Figs. 2 and 3. Any ASM was more efficacious than placebo in the achievement of $\geq 50 \%$ reduction in baseline seizure frequency, and CNB was associated with a higher responder rate than BRV (OR 2.02, 95\% CI 1.11-3.66), ESL (OR 1.93, 95\% CI 1.07-3.48), LCM
(OR 1.86, 95\% CI 1.04-3.32), and PER (OR 2.07, 95\% CI 1.16-3.70) (Fig. 2).

In the analysis by dosages (Table 3), CNB $400 \mathrm{mg} /$ day was associated with a higher responder rate than ESL 800 mg/day (OR 2.65, 95\% CI 1.35-5.22), ESL $1200 \mathrm{mg} /$ day (OR 1.99, 95\% CI 1.03-3.90), BRV 50 mg/day (OR 2.84, 95\% CI 1.34-6.03), BRV $100 \mathrm{mg} /$ day (OR 2.20, 95\% CI 1.10-4.40), BRV 200 mg/day (OR 2.34, 95\% CI 1.15-4.79), LCM 200 mg/day (OR 2.72, 95\% CI 1.38-5.34), PER 4 mg/ day (OR 3.75, 95\% CI 1.87-7.51), PER 8 mg/day (OR 2.31, 95\% CI 1.19-4.48), and PER $12 \mathrm{mg} /$ day (OR 2.01, 95\% CI 1.02-3.96). Cenobamate $200 \mathrm{mg}$ /day was associated with a higher responder rate than PER $4 \mathrm{mg} /$ day (OR 2.67, 95\% CI 1.35-5.29). Lacosamide $400 \mathrm{mg} /$ day was associated with a higher seizure responder rate that LCM $200 \mathrm{mg} /$ day (OR 1.42 , 95\% CI 1.09-1.85), ESL $1200 \mathrm{mg} /$ day was associated with a higher seizure responder rate than ESL $800 \mathrm{mg} /$ day (OR 1.34, 95\% CI 1.03-1.74), and PER $8 \mathrm{mg} /$ day (OR $1.63,95 \% \mathrm{CI} 1.19-2.22$ ) and $12 \mathrm{mg} /$ day (OR $1.86,95 \% \mathrm{CI}$ 

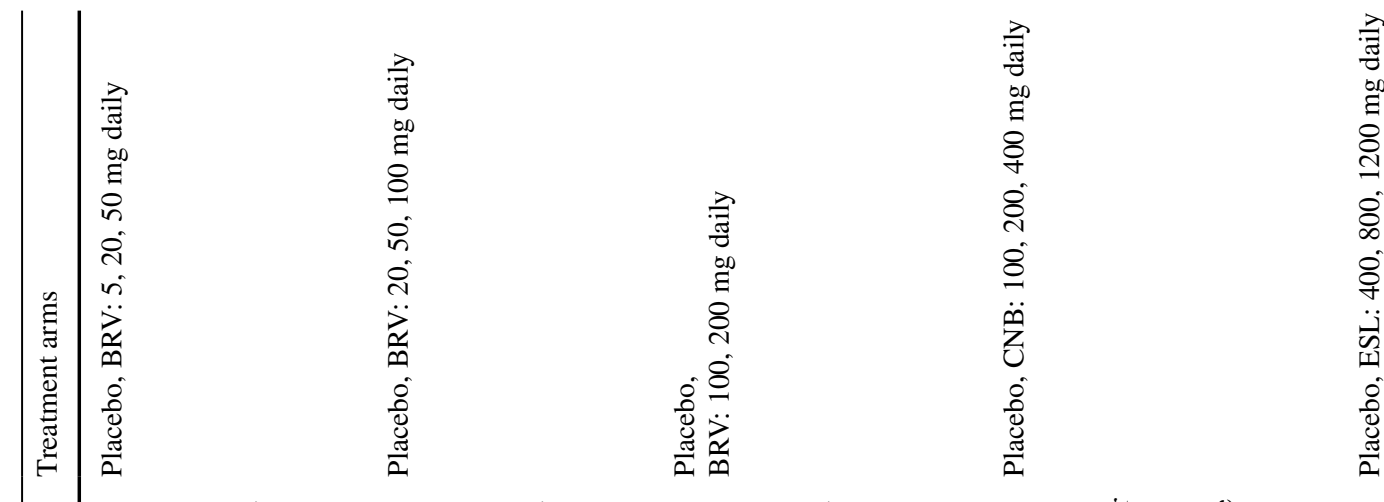

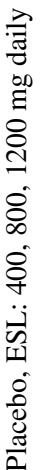

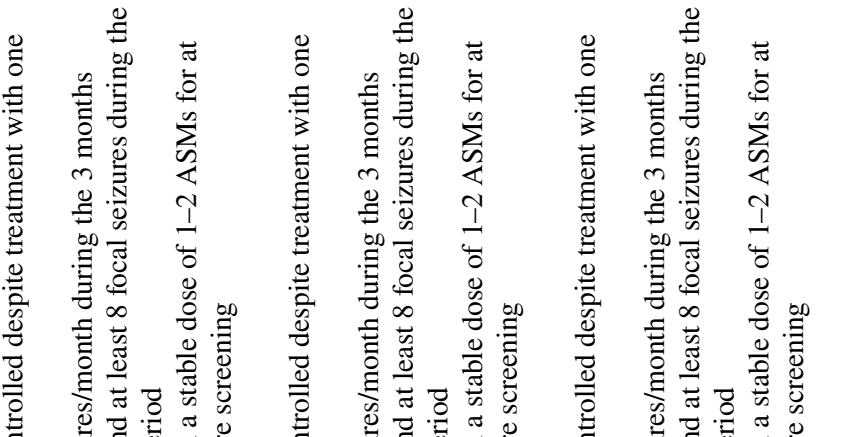

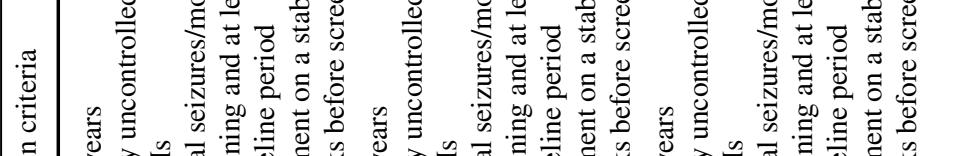

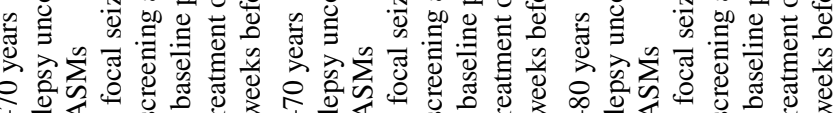
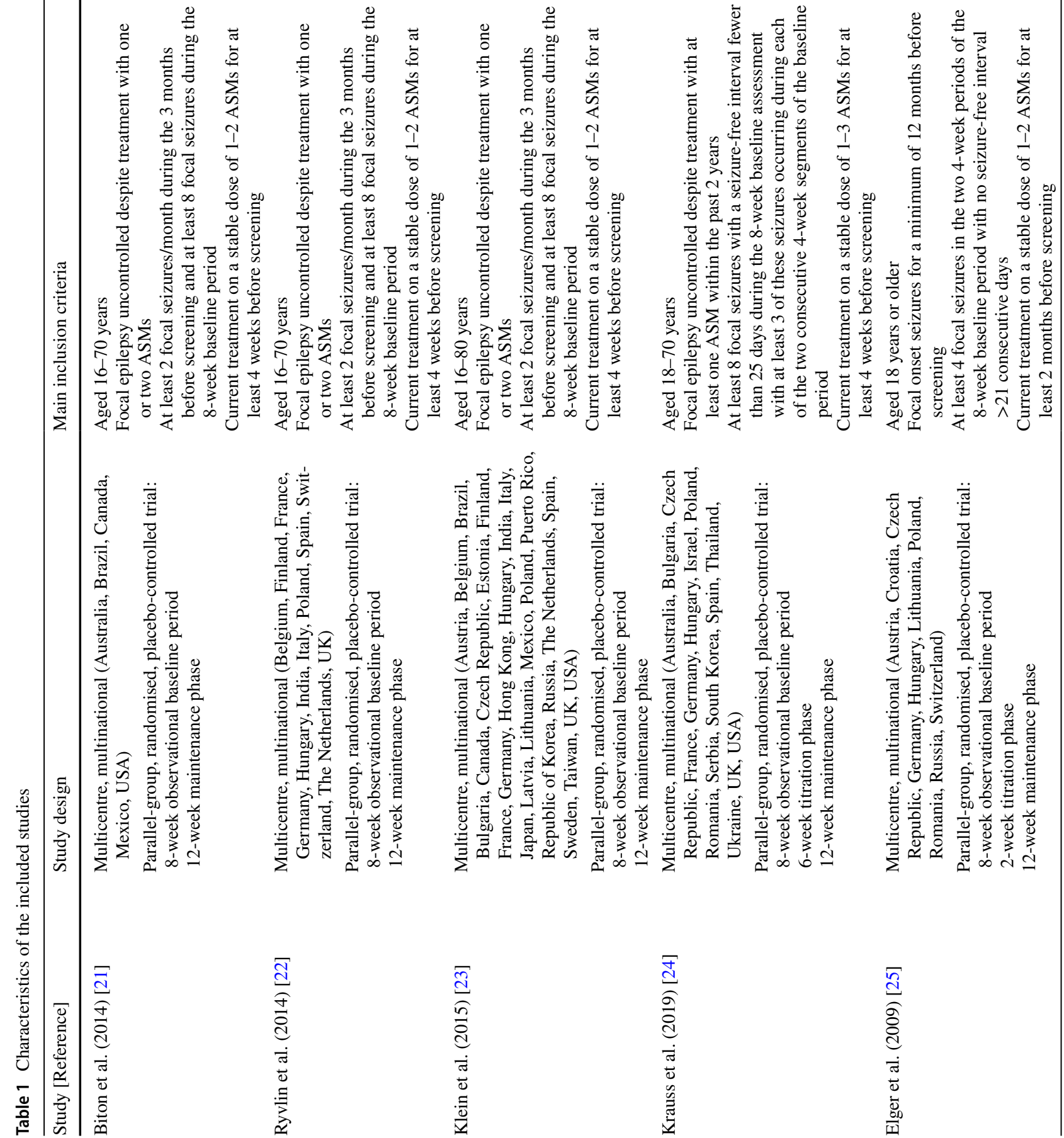

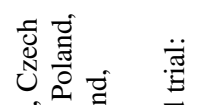

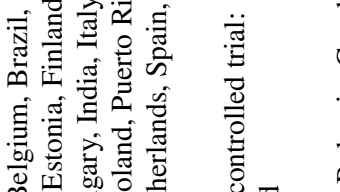

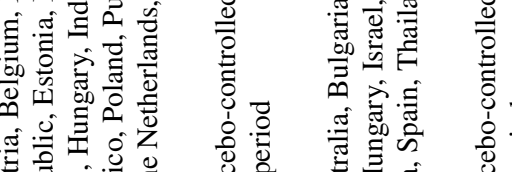

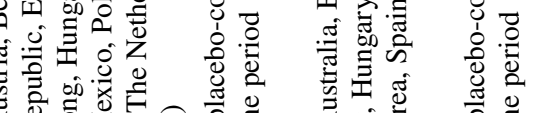

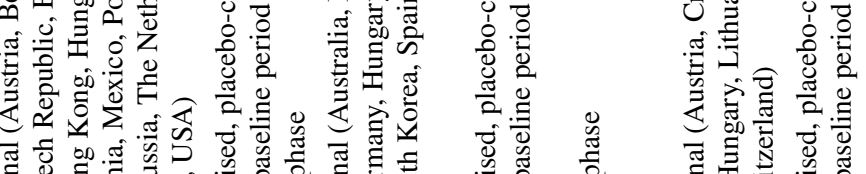

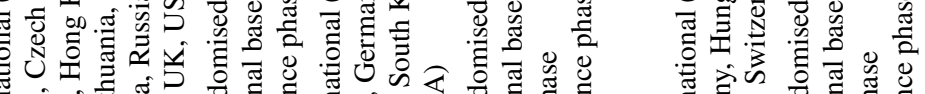

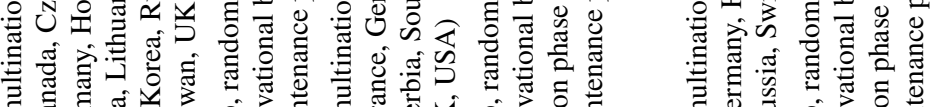

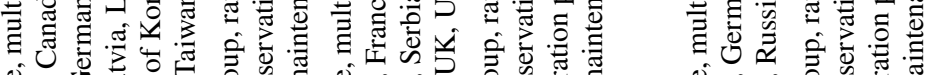

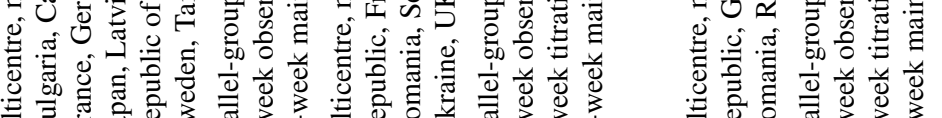

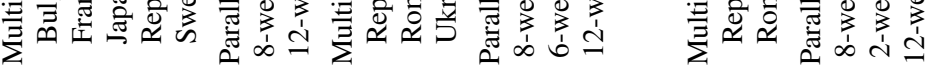

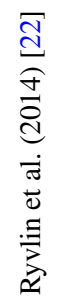

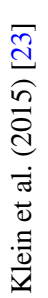

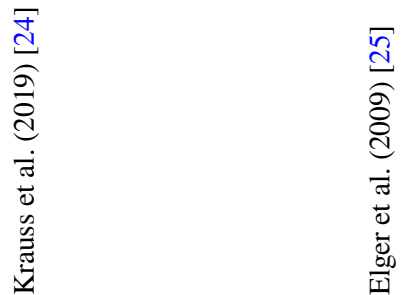

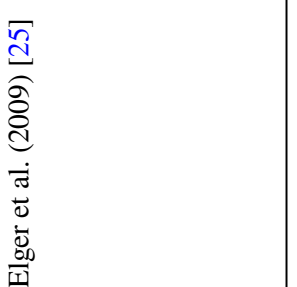




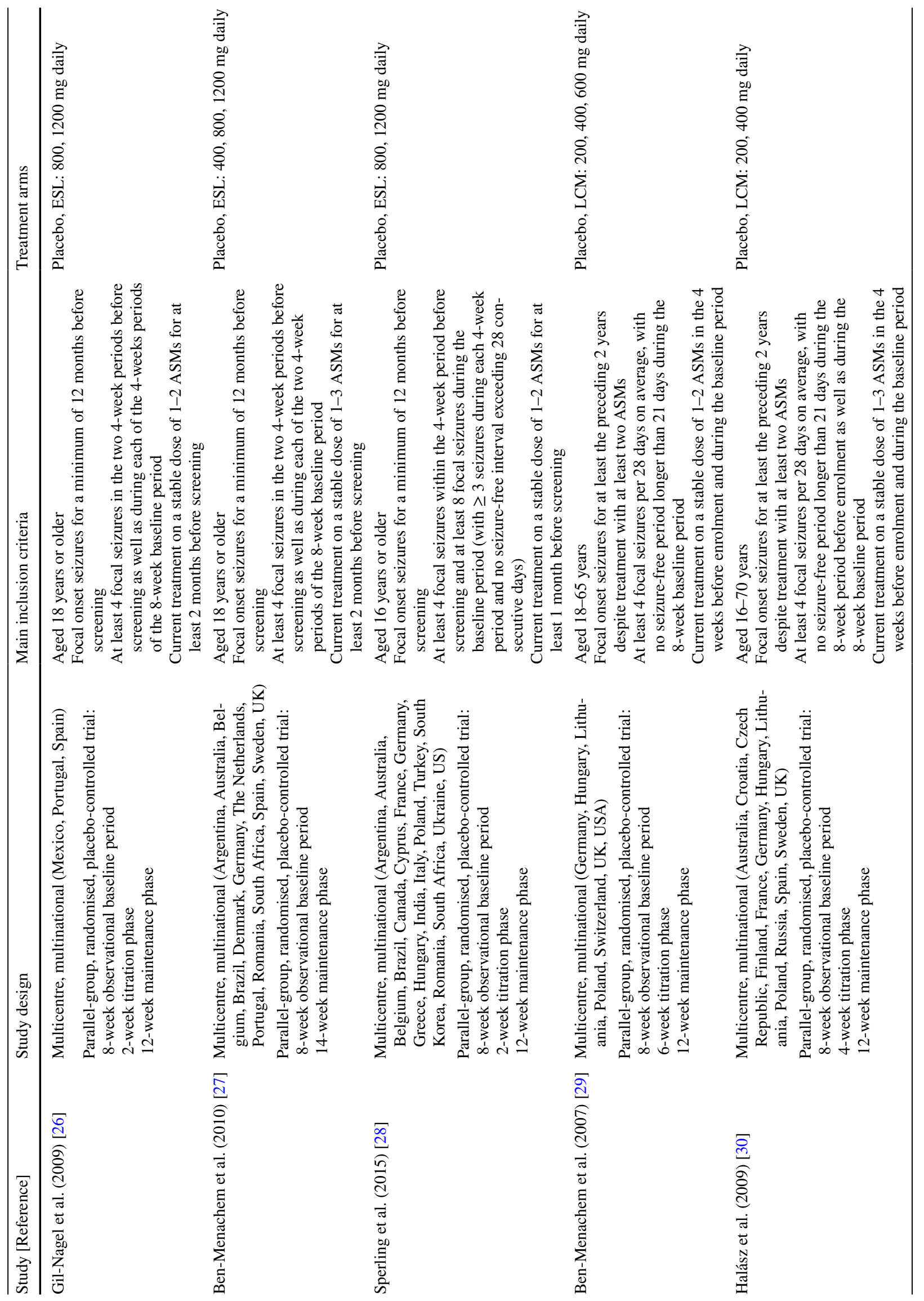




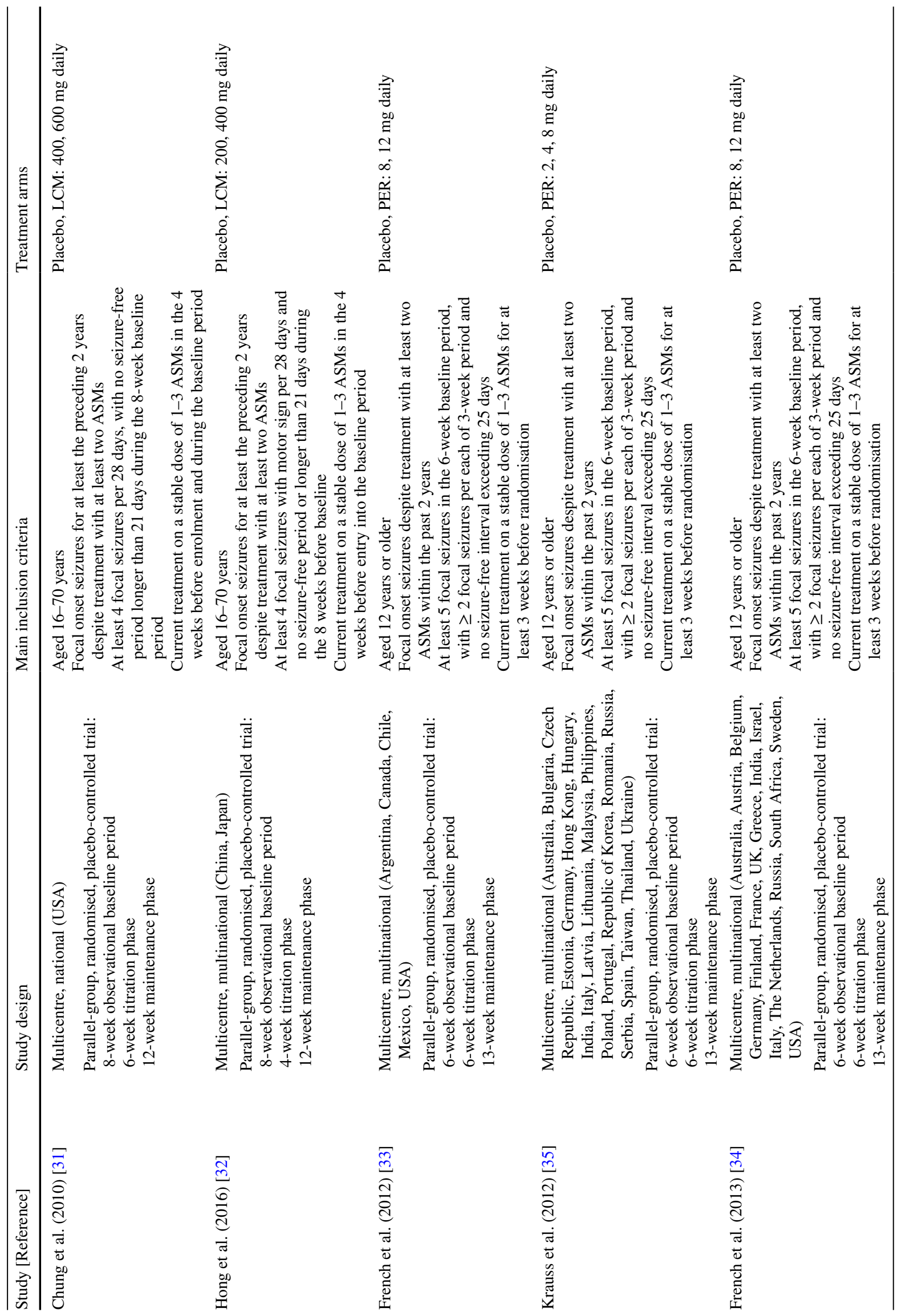


1.32-2.63) was associated with a higher rate of $\geq 50 \%$ seizure frequency reduction than PER $4 \mathrm{mg} /$ day. Perampanel at the daily dosage of $4 \mathrm{mg}$ was associated with a lower rate of seizure responder than ESL $1200 \mathrm{mg} /$ day (OR 0.53, 95\% CI 0.34-0.82), BRV $100 \mathrm{mg} /$ day (OR 0.59, 95\% CI 0.37-0.93), and LCM $400 \mathrm{mg} /$ day (OR 0.51, 95\% CI 0.34-0.78).

With regard the seizure freedom outcome, the network meta-analyses showed BRV, CNB, ESL and PER as being more efficacious than placebo at a statistically significant level (Fig. 3). In the analysis by daily dosages (Table 4), there were no statistically significant differences between the ASMs.

According to SUCRA, CNB had the greatest likelihood ranking best for both seizure response and seizure freedom (Table 5, Appendix II in the SI); in the analysis by drug dosages, CNB $400 \mathrm{mg} /$ day was the option associated with the highest probability to be the most efficacious treatment (Table e-5 and Appendix III in the SI). Results from the loop-specific approach for efficacy outcomes are shown in Appendix IV, and there was no statistically significant incoherence in any loop. Confidence in the evidence for the efficacy outcomes is summarised in Appendix V in the SI.

\subsection{Tolerability Outcomes}

Fourteen RCTs provided data on the proportions of participants who experienced at least one TEAE, whereas RCTs two did not [30, 31]. All RCTs except one LCM study [31] provided data on the proportion of participants who experienced at least one TEAE leading to discontinuation. Figure e-1 in the SI shows the network plots of treatment comparisons for the tolerability and safety outcomes; the corresponding network plots according to treatment dosages are shown in Fig. e-2 in the SI.

At the pairwise meta-analyses, BRV, CNB, ESL, and PER were associated with a higher proportion of patients experiencing at least one TEAE compared to placebo, and the risk of TEAEs leading to treatment discontinuation was higher with any ASM in comparison to placebo (Table e-3 in the SI). In the analysis by dosages, the rate of patients experiencing TEAEs was higher with CNB $400 \mathrm{mg} /$ day than with CNB $200 \mathrm{mg} /$ day, ESL $1200 \mathrm{mg} /$ day than with ESL 800, LCM $400 \mathrm{mg} /$ day than with LCM $200 \mathrm{mg} /$ day, PER $12 \mathrm{mg} /$ day than with PER $4 \mathrm{mg} /$ day, and PER $12 \mathrm{mg} /$ day than with PER $8 \mathrm{mg} /$ day, and the rate of patients experiencing at least one TEAE leading to discontinuation was higher with ESL $1200 \mathrm{mg} /$ day than with ESL 800, LCM $400 \mathrm{mg} /$ day than with LCM $200 \mathrm{mg} /$ day, PER $8 \mathrm{mg}$ /day than with PER $4 \mathrm{mg} /$ day, PER $12 \mathrm{mg} /$ day than with PER $4 \mathrm{mg} /$ day, and PER 12 $\mathrm{mg} /$ day than with PER $8 \mathrm{mg} /$ day (Table e-4 in the SI).

Results of the network meta-analyses of tolerability outcomes are shown in Figs. 4 and 5. Any ASM was associated with a higher rate of patients who experienced at least one 
Table 2 Characteristics of the study participants

\begin{tabular}{|c|c|c|c|c|c|c|c|c|c|}
\hline \multirow[t]{2}{*}{ Study } & \multirow{2}{*}{$\begin{array}{l}\text { Treatment } \\
\text { Arm }\end{array}$} & \multirow{2}{*}{$\begin{array}{l}\text { Number of } \\
\text { participants }\end{array}$} & \multirow[t]{2}{*}{ Age, years } & \multirow{2}{*}{$\begin{array}{l}\text { Male sex, } n \\
(\%)\end{array}$} & \multirow{2}{*}{$\begin{array}{l}\text { Epilepsy dura- } \\
\text { tion, } \\
\text { years }\end{array}$} & \multicolumn{3}{|c|}{ Number of concomitant ASMs, $n(\%)$} & \multirow{2}{*}{$\begin{array}{l}\text { Baseline seizure fre- } \\
\text { quency per } 28 \text { days }\end{array}$} \\
\hline & & & & & & One & Two & Three or more & \\
\hline \multirow{2}{*}{$\begin{array}{l}\text { Biton et al. } \\
\text { (2014) [21] }\end{array}$} & $\mathrm{PBO}$ & 98 & $37.5(12.6)$ & $43(43.9)$ & $24.3(12.2)$ & $13(13.3)$ & $80(81.6)$ & $4(4.1)$ & $2.6[1.6-4.5]^{* *}$ \\
\hline & BRV50 & 101 & $38.9(12.3)$ & $51(50.5)$ & $26.2(12.0)$ & $13(12.9)$ & $82(81.2)$ & $6(5.9)$ & $2.9[1.5-7.2]^{* *}$ \\
\hline \multirow{3}{*}{$\begin{array}{l}\text { Ryvlin et al. } \\
\text { (2014) [22] }\end{array}$} & PBO & 100 & $36.4(13.0)$ & $54(54.0)$ & $20.4(12.3)$ & $14(14.0)$ & $83(83.0)$ & $3(3.0)$ & $2.07[1.35-6.04]^{* *}$ \\
\hline & BRV50 & 99 & $38.9(13.6)$ & $54(54.5)$ & $22.3(13.0)$ & $20(20.2)$ & $77(77.8)$ & $2(2.0)$ & $1.80[1.25-3.47]^{* *}$ \\
\hline & BRV100 & 100 & $38.0(13.1)$ & $58(58.0)$ & $22.1(12.8)$ & $16(16.0)$ & $77(77.0)$ & $7(7.0)$ & $2.02[1.26-3.25]^{* *}$ \\
\hline \multirow{3}{*}{$\begin{array}{l}\text { Klein et al. } \\
\text { (2015) [23] }\end{array}$} & $\mathrm{PBO}$ & 261 & $39.8(12.5)$ & $133(50.1)$ & $22.7(13.3)$ & $14(14.0)$ & $83(83.0)$ & $3(3.0)$ & $10.0(3,560)$ \\
\hline & BRV100 & 253 & $39.1(13.4)$ & $102(40.3)$ & $22.2(13.3)$ & $20(20.2)$ & $77(77.8)$ & $2(2.0)$ & $9.5(2,354)$ \\
\hline & BRV200 & 250 & $39.8(12.8)$ & $133(53.2)$ & $23.4(14.6)$ & $16(16.0)$ & $77(77.0)$ & $7(7.0)$ & $9.3(3,710)$ \\
\hline \multirow{3}{*}{$\begin{array}{l}\text { Krauss et al. } \\
\text { (2019) [24] }\end{array}$} & $\mathrm{PBO}$ & 108 & $39.6(12.4)$ & $58(53.7)$ & $23.0(14.2)$ & $27(25.0)$ & $54(50.0)$ & $27(25.0)$ & $8.4[6.0-19.0$ \\
\hline & CNB200 & 110 & $40.9(12.4)$ & $54(49.1)$ & $22.8(13.2)$ & $39(35.5)$ & $47(42.7)$ & $24(21.8)$ & $11.0[6.0-26.0]$ \\
\hline & CNB400 & 111 & $39.6(10.3)$ & $52(46.8)$ & $24.4(14.2)$ & 24 (21.6) & $62(55.9)$ & $25(22.5)$ & $9.0[6.0-21.5]$ \\
\hline \multirow{3}{*}{$\begin{array}{l}\text { Elger et al. } \\
\text { (2009) [25] }\end{array}$} & PBO & 102 & $37.0(11.9)$ & $48(47.1)$ & $19.4(12.6)$ & $34(33.3)$ & $67(65.7)$ & $1(1.0)$ & $12.4(17.9)$ \\
\hline & ESL800 & 98 & $41.3(12.0)$ & $54(55.1)$ & $23.1(13.5)$ & 31 (31.6) & $67(68.4)$ & 0 & $11.2(11.2)$ \\
\hline & ESL1200 & 102 & 38.4 (11.7) & $44(43.1)$ & 20.4 (11.9) & $39(38.2)$ & $63(61.8)$ & 0 & $11.6(15.9)$ \\
\hline \multirow{3}{*}{$\begin{array}{l}\text { Gil-Nagel } \\
\text { et al. (2009) } \\
{[26]}\end{array}$} & PBO & 87 & $37.7(12.1)$ & $43(49.4)$ & $23.8(13.0)$ & $16(18.4)$ & $66(75.9)$ & $5(5.7)$ & $11.3(18.5)$ \\
\hline & ESL800 & 85 & $36.8(10.7)$ & $35(41.2)$ & $22.5(11.8)$ & $22(25.9)$ & $58(68.2)$ & $5(5.9)$ & $11.6(22.1)$ \\
\hline & ESL1200 & 80 & $36.0(11.4)$ & $35(43.8)$ & $23.0(13.0)$ & $12(15.0)$ & $63(78.8)$ & $5(6.3)$ & $11.3(10.3)$ \\
\hline \multirow{3}{*}{$\begin{array}{l}\text { Ben-Mena- } \\
\text { chem et al. } \\
(2010) \text { [27] }\end{array}$} & $\mathrm{PBO}$ & 100 & $36.7(12.2)$ & $52(52.0)$ & $25.4(13.1)$ & $15(15.0)$ & $76(76.0)$ & $9(9.0)$ & $14.3(16.6)$ \\
\hline & ESL800 & 101 & 36.4 (12.6) & $51(50.5)$ & 22.4 (11.6) & $17(16.8)$ & $73(72.3)$ & $11(10.9)$ & $16.5(19.6)$ \\
\hline & ESL1200 & 98 & 36.9 (11.6) & $52(53.1)$ & $23.0(12.9)$ & $20(20.4)$ & $68(69.4)$ & $10(10.2)$ & $14.8(16.0)$ \\
\hline \multirow{3}{*}{$\begin{array}{l}\text { Sperling et al. } \\
\text { (2015) [28] }\end{array}$} & PBO & 224 & $39.0(16,67)$ & $112(50.0)$ & $21.3(14.6)$ & 64 (28.6) & $158(70.5)$ & $1(0.4)$ & $8(4,282)$ \\
\hline & ESL800 & 216 & $38.5(16,71)$ & $109(50.5)$ & $21.6(13.0)$ & $60(27.8)$ & $153(70.8)$ & 0 & $8(1,420)$ \\
\hline & ESL1200 & 210 & $38.0(16,69)$ & $105(50.0)$ & $21.2(13.0)$ & $59(28.1)$ & 151 (71.9) & 0 & $9(4,351)$ \\
\hline $\begin{array}{l}\text { Ben-Mena- } \\
\text { chem et al. } \\
\text { (2007) [29] }\end{array}$ & $\begin{array}{l}\text { PBO } \\
\text { LCM200 } \\
\text { LCM } 400\end{array}$ & $\begin{array}{l}97 \\
107 \\
108\end{array}$ & $\begin{array}{l}38.9(11.1) \\
39.9(11.7) \\
41.2(11.6)\end{array}$ & $\begin{array}{l}47(48.5) \\
46(43.0) \\
53(49.1)\end{array}$ & $\begin{array}{l}24.6(11.8) \\
25.1(12.9) \\
24.7(13.1)\end{array}$ & \multicolumn{3}{|c|}{$\begin{array}{l}16 \% \text { and } 84 \% \text { of the patients were taking } \\
1 \text { and } 2 \text { ASMs }\end{array}$} & $\begin{array}{l}\text { Median seizure } \\
\text { frequency ranged } \\
\text { from } 11 \text { to } 13 \text { across } \\
\text { all arms }\end{array}$ \\
\hline \multirow{3}{*}{$\begin{array}{l}\text { Halász et al. } \\
\text { (2009) [30] }\end{array}$} & PBO & 163 & 38.5 (10.9) & $91(55.8)$ & $21.1(12.2)$ & $21(13.2)$ & $82(51.6)$ & $56(35.2)$ & $9.9 *$ \\
\hline & LCM200 & 163 & $36.9(11.7)$ & $90(55.2)$ & $22.9(12.3)$ & $17(10.6)$ & $77(48.1)$ & $66(41.3)$ & $11.5^{*}$ \\
\hline & LCM400 & 159 & $37.9(13.0)$ & $69(43.4)$ & $22.8(13.2)$ & $25(15.8)$ & $79(50.0)$ & $54(34.2)$ & $10.3 *$ \\
\hline \multirow{2}{*}{$\begin{array}{l}\text { Chung et al. } \\
\text { (2010) [31] }\end{array}$} & РBO & 104 & $38.1(12.0)$ & $49(47.1)$ & $25.4(13.3)$ & $18(17.3)$ & $54(51.9)$ & $32(30.8)$ & $15.0^{*}$ \\
\hline & LCM400 & 204 & $39.1(12.4)$ & $104(51.0)$ & $24.5(13.2)$ & $36(17.9)$ & $110(54.7)$ & $55(27.4)$ & $11.5^{*}$ \\
\hline \multirow{3}{*}{$\begin{array}{l}\text { Hong et al. } \\
\text { (2016) [32] }\end{array}$} & PBO & 184 & $31.8(12.0)$ & $102(55.4)$ & $16.8(11.5)$ & $41(22.4)$ & $71(38.8)$ & $71(38.8)$ & $10.5(3.6,707.6)$ \\
\hline & LCM200 & 183 & $33.2(12.2)$ & $94(51.4)$ & $18.3(10.9)$ & $45(24.7)$ & $79(43.4)$ & $58(31.9)$ & $11.0(3.7,1118.0)$ \\
\hline & LCM400 & 180 & $32.3(11.9)$ & $104(57.8)$ & $17.9(11.7)$ & 35 (19.6) & $81(45.3)$ & $63(35.2)$ & $10.0(2.6,221.0)$ \\
\hline \multirow{3}{*}{$\begin{array}{l}\text { French et al. } \\
\text { (2012) [33] }\end{array}$} & РBO & 121 & $35.6(14.7)$ & $54(44.6)$ & $24.1(12.9)^{\S}$ & $15(12.4)$ & $64(52.9)$ & $42(34.7)$ & $13.7(3.3,227.4)$ \\
\hline & PER8 & 133 & $35.8(14.2)$ & $65(48.9)$ & $23.6(13.5)^{\S}$ & $26(19.5)$ & $70(52.9)$ & $37(27.8)$ & $14.3(2.4,1030.8)$ \\
\hline & PER12 & 134 & 36.7 (14.6) & $69(51.5)$ & $23.3(14.4)^{\S}$ & $19(14.2)$ & $82(61.2)$ & $33(24.6)$ & $12.0(2.9,1083.1)$ \\
\hline Kra & PBO & 185 & 33.4 (12.6) & $95(51.4)$ & $17.5(10.7)^{\S}$ & $28(15.1)$ & $90(48.6)$ & $67(36.2)$ & $9.3(3.3,569.1)$ \\
\hline & PER4 & 172 & $33.6(12.2)$ & $88(51.2)$ & $19.7(12.1)^{\S}$ & $19(11.0)$ & $88(51.2)$ & $65(37.8)$ & $10.0(2.9,4503.9)$ \\
\hline & PER8 & 169 & $34.6(12.8)$ & 77 (45.6) & $20.0(11.9)^{\S}$ & $27(16.0)$ & $82(48.5)$ & $60(35.5)$ & $10.9(3.4,723.2)$ \\
\hline $\begin{array}{l}\text { French et al. } \\
\text { (2013) [34] }\end{array}$ & $\begin{array}{l}\text { PBO } \\
\text { PER8 }\end{array}$ & $\begin{array}{l}136 \\
129\end{array}$ & $\begin{array}{l}34.4(13.6) \\
36.7(14.4)\end{array}$ & $\begin{array}{l}71(52.2) \\
65(50.4)\end{array}$ & $\begin{array}{l}22.0(12.9)^{\S} \\
22.5(13.6)^{\S}\end{array}$ & $\begin{array}{l}17(12.5) \\
16(12.4)\end{array}$ & $\begin{array}{l}64(47.1) \\
68(52.7)\end{array}$ & $\begin{array}{l}71(52.2) \\
83(64.3)\end{array}$ & $11.8(3.4,358.4)$ \\
\hline & PER12 & 121 & $35.5(14.1)$ & $50(41.3)$ & $21.3(13.2)^{\S}$ & $9(7.4)$ & $63(52.1)$ & $80(66.1)$ & $13.7(1.4,598.4)$ \\
\hline Nishida et al. & PBO & 175 & $34.5(13.2)$ & $86(49.1)$ & $17.5(10.9)$ & $11(6.3)$ & $67(38.3)$ & $97(55.4)$ & Median seizure fre- \\
\hline & PER8 & 175 & $33.6(14.1)$ & $91(52.0)$ & $16.9(11.5)$ & $15(8.6)$ & $60(34.3)$ & $100(57.1)$ & 19 \\
\hline & PER12 & 180 & $32.3(12.3)$ & $87(48.3)$ & $17.4(11.2)$ & $13(7.2)$ & 75 (41.7) & $92(51.1)$ & all arms \\
\hline
\end{tabular}

Data are mean (SD), median [IQR] or median (min, max) unless otherwise specified. *Median. **Baseline seizure frequency per week. ${ }^{\S}$ Converted to years by dividing the number of months by 12

$A S M$ antiseizure medication, $B R V$ brivaracetam, $C N B$ cenobamate, $E S L$ eslicarbazepine acetate, IQR interquartile range, $L C M$ lacosamide, $P E R$ perampanel, $S D$ standard deviation 
TEAE in comparison to placebo; BRV (OR 0.61, 95\% CI $0.44-0.86$ ) and LCM (OR $0.60,95 \%$ CI $0.40-0.88$ ) were associated with a lower proportion of participants experiencing TEAEs in comparison to ESL, and PER was associated with a higher risk of experiencing at least one TEAE (OR 1.42, 95\% CI 1.02-1.96) than BRV (Fig. 4). Any ASM was associated with a higher rate of patients who experienced at least one TEAE leading to discontinuation than placebo, whereas there were no statistically significant differences across the individual ASMs (Fig. 5).

In the analysis by dosages (Table 6), the rate of patients who experienced at least one TEAE was higher with ESL $1200 \mathrm{mg} /$ day than with ESL $800 \mathrm{mg} /$ day (OR 1.43, 95\% CI $1.09-1.88$ ) and PER $12 \mathrm{mg} /$ day than with ESL $800 \mathrm{mg} /$ day (OR 1.66, 95\% CI 1.08-2.56), while it was lower with LCM $200 \mathrm{mg} /$ day compared to ESL $800 \mathrm{mg} /$ day (OR $0.55,95 \%$ CI 0.35-0.86). In comparison to ESL $1200 \mathrm{mg} / \mathrm{day}$, BRV $50 \mathrm{mg} /$ day (OR 0.45, 95\% CI 0.28-4.61), BRV $100 \mathrm{mg} /$ day (OR $0.55,95 \%$ CI 0.37-0.82), BRV $200 \mathrm{mg} /$ day (OR 0.52, 95\% CI 0.33-0.80), LCM $200 \mathrm{mg}$ /day (OR 0.39, 95\% CI $0.25-0.61$ ), and PER $4 \mathrm{mg} / \mathrm{day}$ (OR $0.50,95 \%$ CI $0.34-0.75$ ) were associated with lower proportions of patients who experienced at least one TEAE. Compared to BRV $50 \mathrm{mg} /$ day, PER $12 \mathrm{mg} /$ day (OR 2.56, 95\% CI 1.50-4.38) and CNB $400 \mathrm{mg} /$ day (OR 3.23, 95\% CI 1.38-7.56) showed a high risk of TEAE occurrence. Perampanel $12 \mathrm{mg} /$ day and CNB $400 \mathrm{mg} / \mathrm{day}$ were associated with a higher rate of patients who experienced at least one TEAE compared to BRV $100 \mathrm{mg} /$ day (PER $12 \mathrm{mg}$ /day: OR 2.12, 95\% CI 1.34-3.35; CNB $400 \mathrm{mg} /$ day: OR 2.67, 95\% CI 1.19-5.97) and BRV $200 \mathrm{mg} /$ day (PER $12 \mathrm{mg} /$ day: OR 2.24, 95\% CI 1.37-3.66; CNB 400 mg/day: OR 2.82, 95\% CI 1.24-6.44).

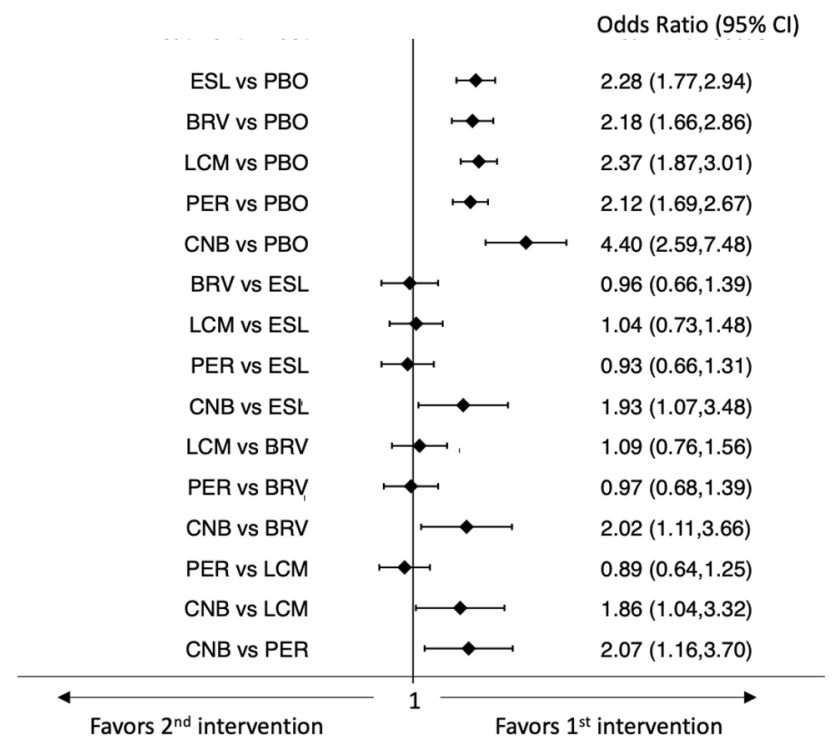

Fig. 2 Interval plot for the efficacy outcome: seizure response. $B R V$ brivaracetam, $C N B$ cenobamate, $C I$ confidence interval, $E S L$ eslicarbazepine acetate, $L C M$ lacosamide, $P B O$ placebo, $P E R$ perampanel
The rates of patients experiencing TEAEs were higher with LCM $400 \mathrm{mg} /$ day (OR 1.68, 95\% CI 1.15-2.48), PER $8 \mathrm{mg} /$ day (OR 1.85, 95\% CI 1.18-2.89), PER $12 \mathrm{mg} /$ day (OR 3.00, 95\% CI 1.82-4.94), and CNB $400 \mathrm{mg} /$ day (OR $3.77,95 \%$ CI 1.65-8.65) in comparison to LCM $200 \mathrm{mg} /$ day. The rate of patients with at least one TEAE was higher with PER $8 \mathrm{mg} /$ day than PER $4 \mathrm{mg} /$ day (OR 1.42, 95\% CI 1.04-1.93), and with PER $12 \mathrm{mg} /$ day than with PER 4 $\mathrm{mg}$ /day (OR 2.30, 95\% CI 1.55-3.43), PER $8 \mathrm{mg}$ /day (OR $1.62,95 \%$ CI 1.13-2.33), and LCM $400 \mathrm{mg} /$ day (OR 1.78, 95\% CI 1.06-2.99). Cenobamate $400 \mathrm{mg} /$ day was associated with a statistically significantly higher rate of patients with at least one TEAE than PER $4 \mathrm{mg}$ /day (OR 2.90, 95\% CI 1.30-6.48). Further, CNB $200 \mathrm{mg} /$ day was associated with a lower rate of patients who experienced at least one TEAE compared to PER 12 (OR 0.45, 95\% CI 0.22-0.90), and CNB $400 \mathrm{mg} / \mathrm{day}$ was associated with a higher rate of patients who experienced at least one TEAE compared to CNB 200 mg/day (OR 2.81, 95\% CI 1.31-6.03).

Concerning the proportion of patients who experienced at least one TEAE leading to discontinuation according to daily dosages (Table 7), the rates were higher with ESL 1200 $\mathrm{mg} /$ day (OR 2.11, 95\% CI 1.49-2.98) and PER $12 \mathrm{mg} /$ day (OR 2.07, 95\% CI 1.07-4.02). Compared with ESL 1200 $\mathrm{mg} / \mathrm{day}$, BRV $200 \mathrm{mg} / \mathrm{day}$ (OR 0.42, 95\% CI 0.17-0.99), LCM $200 \mathrm{mg} /$ day (OR 0.29, 95\% CI 0.14-0.58), PER $4 \mathrm{mg} /$ day (OR 0.23, 95\% CI 0.10-0.52) and PER $8 \mathrm{mg} /$ day (OR $0.51,95 \%$ CI $0.27-0.97$ ) were associated with a lower rate of patients experiencing at least one TEAE leading to discontinuation. The rates of participants experiencing TEAEs

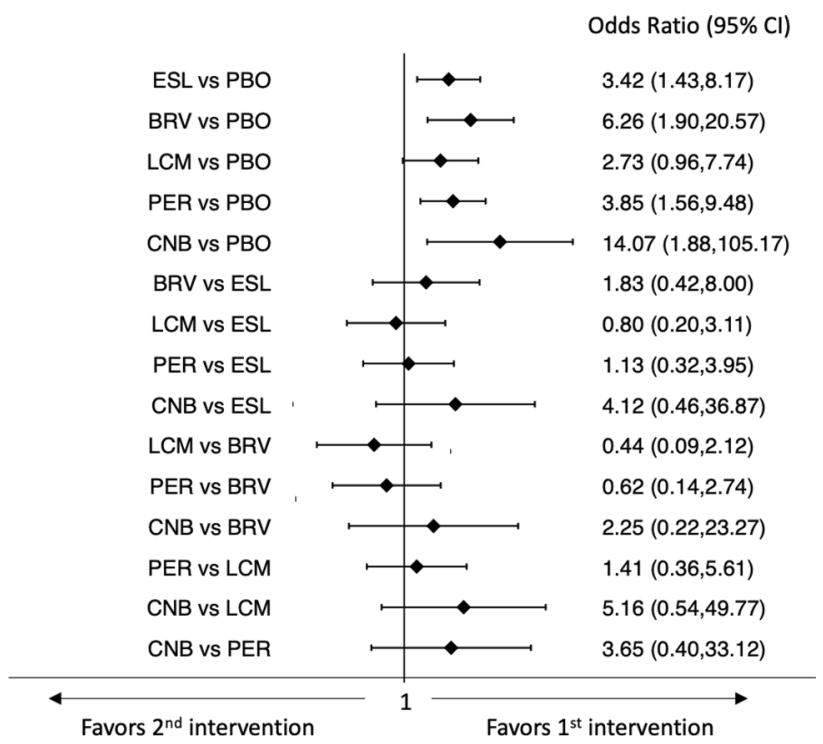

Fig. 3 Interval plot for the efficacy outcome: seizure freedom. $B R V$ brivaracetam, $C N B$ cenobamate, $C I$ confidence interval, $E S L$ eslicarbazepine acetate, $L C M$ lacosamide, $P B O$ placebo, $P E R$ perampanel 
Table 3 Network league table for the responder rate

\begin{tabular}{|c|c|c|c|c|c|c|c|c|c|c|c|c|}
\hline PBO & $\begin{array}{c}1.98 \\
(1.48,2.63)\end{array}$ & $\begin{array}{c}2.64 \\
(1.99,3.51)\end{array}$ & $\begin{array}{c}1.85 \\
(1.19,2.87)\end{array}$ & $\begin{array}{c}2.38 \\
(1.72,3.29)\end{array}$ & $\begin{array}{c}2.24 \\
(1.55,3.25)\end{array}$ & $\begin{array}{c}1.93 \\
(1.45,2.57)\end{array}$ & $\begin{array}{c}2.74 \\
(2.11,3.55)\end{array}$ & $\begin{array}{c}1.40 \\
(1.01,1.94)\end{array}$ & $\begin{array}{c}2.27 \\
(1.75,2.95)\end{array}$ & $\begin{array}{c}2.60 \\
(1.95,3.47)\end{array}$ & $\begin{array}{c}3.74 \\
(2.06,6.80)\end{array}$ & $\begin{array}{c}5.24 \\
(2.84,9.67)\end{array}$ \\
\hline $\begin{array}{c}0.51 \\
(0.38,0.67)\end{array}$ & ESL800 & $\begin{array}{c}1.34 \\
(1.03,1.74)\end{array}$ & $\begin{array}{c}0.93 \\
(0.55,1.58)\end{array}$ & $\begin{array}{c}1.21 \\
(0.78,1.86)\end{array}$ & $\begin{array}{c}1.13 \\
(0.71,1.81)\end{array}$ & $\begin{array}{c}0.98 \\
(0.65,1.47)\end{array}$ & $\begin{array}{c}1.38 \\
(0.94,2.04)\end{array}$ & $\begin{array}{c}0.71 \\
(0.46,1.09)\end{array}$ & $\begin{array}{c}1.15 \\
(0.78,1.70)\end{array}$ & $\begin{array}{c}1.32 \\
(0.88,1.98)\end{array}$ & $\begin{array}{c}1.89 \\
(0.97,3.67)\end{array}$ & $\begin{array}{c}2.65 \\
(1.35,5.22)\end{array}$ \\
\hline $\begin{array}{c}0.38 \\
(0.28,0.50)\end{array}$ & $\begin{array}{c}0.75 \\
(0.58,0.97)\end{array}$ & ESL1200 & $\begin{array}{c}0.70 \\
(0.41,1.18)\end{array}$ & $\begin{array}{c}0.90 \\
(0.59,1.39)\end{array}$ & $\begin{array}{c}0.85 \\
(0.53,1.35)\end{array}$ & $\begin{array}{c}0.73 \\
(0.49,1.09)\end{array}$ & $\begin{array}{c}1.04 \\
(0.70,1.52)\end{array}$ & $\begin{array}{c}0.53 \\
(0.34,0.82)\end{array}$ & $\begin{array}{c}0.86 \\
(0.59,1.27)\end{array}$ & $\begin{array}{c}0.99 \\
(0.66,1.48)\end{array}$ & $\begin{array}{c}1.42 \\
(0.73,2.74)\end{array}$ & $\begin{array}{c}1.99 \\
(1.01,3.90)\end{array}$ \\
\hline $\begin{array}{c}0.54 \\
(0.35,0.84)\end{array}$ & $\begin{array}{c}1.07 \\
(0.63,1.81)\end{array}$ & $\begin{array}{c}1.43 \\
(0.85,2.42)\end{array}$ & BRV50 & $\begin{array}{c}1.29 \\
(0.80,2.07)\end{array}$ & $\begin{array}{c}1.21 \\
(0.71,2.07)\end{array}$ & $\begin{array}{c}1.05 \\
(0.62,1.77)\end{array}$ & $\begin{array}{c}1.48 \\
(0.89,2.47)\end{array}$ & $\begin{array}{c}0.76 \\
(0.44,1.31)\end{array}$ & $\begin{array}{c}1.23 \\
(0.74,2.05)\end{array}$ & $\begin{array}{c}1.41 \\
(0.83,2.39)\end{array}$ & $\begin{array}{c}2.02 \\
(0.96,4.25)\end{array}$ & $\begin{array}{c}2.84 \\
(1.34,6.03)\end{array}$ \\
\hline $\begin{array}{c}0.42 \\
(0.30,0.58)\end{array}$ & $\begin{array}{c}0.83 \\
(0.54,1.28)\end{array}$ & $\begin{array}{c}1.11 \\
(0.72,1.71)\end{array}$ & $\begin{array}{c}0.78 \\
(0.48,1.24)\end{array}$ & BRV100 & $\begin{array}{c}0.94 \\
(0.66,1.33)\end{array}$ & $\begin{array}{c}0.81 \\
(0.53,1.25)\end{array}$ & $\begin{array}{c}1.15 \\
(0.76,1.74)\end{array}$ & $\begin{array}{c}0.59 \\
(0.37,0.93)\end{array}$ & $\begin{array}{c}0.96 \\
(0.63,1.45)\end{array}$ & $\begin{array}{c}1.09 \\
(0.71,1.69)\end{array}$ & $\begin{array}{c}1.57 \\
(0.80,3.10)\end{array}$ & $\begin{array}{c}2.20 \\
(1.10,4.40)\end{array}$ \\
\hline $\begin{array}{c}0.45 \\
(0.31,0.65)\end{array}$ & $\begin{array}{c}0.88 \\
(0.55,1.41)\end{array}$ & $\begin{array}{c}1.18 \\
(0.74,1.88)\end{array}$ & $\begin{array}{c}0.82 \\
(0.48,1.41)\end{array}$ & $\begin{array}{c}1.06 \\
(0.75,1.51)\end{array}$ & BRV200 & $\begin{array}{c}0.86 \\
(0.54,1.38)\end{array}$ & $\begin{array}{c}1.22 \\
(0.78,1.92)\end{array}$ & $\begin{array}{c}0.62 \\
(0.38,1.03)\end{array}$ & $\begin{array}{c}1.02 \\
(0.65,1.60)\end{array}$ & $\begin{array}{c}1.16 \\
(0.73,1.86)\end{array}$ & $\begin{array}{c}1.67 \\
(0.83,3.37)\end{array}$ & $\begin{array}{c}2.34 \\
(1.15,4.79)\end{array}$ \\
\hline $\begin{array}{c}0.52 \\
(0.39,0.69)\end{array}$ & $\begin{array}{c}1.02 \\
(0.68,1.54)\end{array}$ & $\begin{array}{c}1.37 \\
(0.91,2.05)\end{array}$ & $\begin{array}{c}0.96 \\
(0.57,1.62)\end{array}$ & $\begin{array}{c}1.23 \\
(0.80,1.90)\end{array}$ & $\begin{array}{c}1.16 \\
(0.73,1.85)\end{array}$ & LCM200 & $\begin{array}{c}1.42 \\
(1.09,1.85)\end{array}$ & $\begin{array}{c}0.72 \\
(0.47,1.12)\end{array}$ & $\begin{array}{c}1.18 \\
(0.80,1.74)\end{array}$ & $\begin{array}{c}1.35 \\
(0.90,2.03)\end{array}$ & $\begin{array}{c}1.94 \\
(1.00,3.76)\end{array}$ & $\begin{array}{c}2.72 \\
(1.38,5.34)\end{array}$ \\
\hline $\begin{array}{c}0.37 \\
(0.28,0.47)\end{array}$ & $\begin{array}{c}0.72 \\
(0.49,1.06)\end{array}$ & $\begin{array}{c}0.97 \\
(0.66,1.42)\end{array}$ & $\begin{array}{c}0.67 \\
(0.40,1.13)\end{array}$ & $\begin{array}{c}0.87 \\
(0.57,1.32)\end{array}$ & $\begin{array}{c}0.82 \\
(0.52,1.29)\end{array}$ & $\begin{array}{c}0.71 \\
(0.54,0.92)\end{array}$ & LCM400 & $\begin{array}{c}0.51 \\
(0.34,0.78)\end{array}$ & $\begin{array}{c}0.83 \\
(0.57,1.20)\end{array}$ & $\begin{array}{c}0.95 \\
(0.65,1.40)\end{array}$ & $\begin{array}{c}1.37 \\
(0.71,2.62)\end{array}$ & $\begin{array}{c}1.92 \\
(0.99,3.73)\end{array}$ \\
\hline $\begin{array}{c}0.72 \\
(0.51,0.99)\end{array}$ & $\begin{array}{c}1.41 \\
(0.91,2.19)\end{array}$ & $\begin{array}{c}1.89 \\
(1.22,2.92)\end{array}$ & $\begin{array}{c}1.32 \\
(0.76,2.29)\end{array}$ & $\begin{array}{c}1.70 \\
(1.07,2.70)\end{array}$ & $\begin{array}{c}1.60 \\
(0.98,2.63)\end{array}$ & $\begin{array}{c}1.38 \\
(0.89,2.13)\end{array}$ & $\begin{array}{c}1.96 \\
(1.29,2.98)\end{array}$ & PER4 & $\begin{array}{c}1.63 \\
(1.19,2.22)\end{array}$ & $\begin{array}{c}1.86 \\
(1.32,2.63)\end{array}$ & $\begin{array}{c}2.67 \\
(1.35,5.29)\end{array}$ & $\begin{array}{c}3.75 \\
(1.87,7.51)\end{array}$ \\
\hline $\begin{array}{c}0.44 \\
(0.34,0.57)\end{array}$ & $\begin{array}{c}0.87 \\
(0.59,1.28)\end{array}$ & $\begin{array}{c}1.16 \\
(0.79,1.71)\end{array}$ & $\begin{array}{c}0.81 \\
(0.49,1.35)\end{array}$ & $\begin{array}{c}1.05 \\
(0.69,1.59)\end{array}$ & $\begin{array}{c}0.98 \\
(0.63,1.55)\end{array}$ & $\begin{array}{c}0.85 \\
(0.58,1.25)\end{array}$ & $\begin{array}{c}1.20 \\
(0.83,1.74)\end{array}$ & $\begin{array}{c}0.61 \\
(0.45,0.84)\end{array}$ & PER8 & $\begin{array}{c}1.14 \\
(0.88,1.49)\end{array}$ & $\begin{array}{c}1.64 \\
(0.86,3.16)\end{array}$ & $\begin{array}{c}2.31 \\
(1.19,4.48)\end{array}$ \\
\hline $\begin{array}{c}0.38 \\
(0.29,0.51)\end{array}$ & $\begin{array}{c}0.76 \\
(0.51,1.14)\end{array}$ & $\begin{array}{c}1.01 \\
(0.68,1.52)\end{array}$ & $\begin{array}{c}0.71 \\
(0.42,1.20)\end{array}$ & $\begin{array}{c}0.91 \\
(0.59,1.41)\end{array}$ & $\begin{array}{c}0.86 \\
(0.54,1.38)\end{array}$ & $\begin{array}{c}0.74 \\
(0.49,1.11)\end{array}$ & $\begin{array}{c}1.05 \\
(0.71,1.55)\end{array}$ & $\begin{array}{c}0.54 \\
(0.38,0.76)\end{array}$ & $\begin{array}{c}0.87 \\
(0.67,1.14)\end{array}$ & PER12 & $\begin{array}{c}1.44 \\
(0.74,2.79)\end{array}$ & $\begin{array}{c}2.01 \\
(1.02,3.96)\end{array}$ \\
\hline $\begin{array}{c}0.27 \\
(0.15,0.49)\end{array}$ & $\begin{array}{c}0.53 \\
(0.27,1.03)\end{array}$ & $\begin{array}{c}0.71 \\
(0.36,1.37)\end{array}$ & $\begin{array}{c}0.49 \\
(0.24,1.04)\end{array}$ & $\begin{array}{c}0.64 \\
(0.32,1.26)\end{array}$ & $\begin{array}{c}0.60 \\
(0.30,1.21)\end{array}$ & $\begin{array}{c}0.52 \\
(0.27,1.00)\end{array}$ & $\begin{array}{c}0.73 \\
(0.38,1.41)\end{array}$ & $\begin{array}{c}0.37 \\
(0.19,0.74)\end{array}$ & $\begin{array}{c}0.61 \\
(0.32,1.17)\end{array}$ & $\begin{array}{c}0.70 \\
(0.36,1.35)\end{array}$ & CNB200 & $\begin{array}{c}1.40 \\
(0.79,2.50)\end{array}$ \\
\hline $\begin{array}{c}0.19 \\
(0.10,0.35)\end{array}$ & $\begin{array}{c}0.38 \\
(0.19,0.74)\end{array}$ & $\begin{array}{c}0.50 \\
(0.26,0.99)\end{array}$ & $\begin{array}{c}0.35 \\
(0.17,0.75)\end{array}$ & $\begin{array}{c}0.45 \\
(0.23,0.91)\end{array}$ & $\begin{array}{c}0.43 \\
(0.21,0.87)\end{array}$ & $\begin{array}{c}0.37 \\
(0.19,0.72)\end{array}$ & $\begin{array}{c}0.52 \\
(0.27,1.01)\end{array}$ & $\begin{array}{c}0.27 \\
(0.13,0.53)\end{array}$ & $\begin{array}{c}0.43 \\
(0.22,0.84)\end{array}$ & $\begin{array}{c}0.50 \\
(0.25,0.98)\end{array}$ & $\begin{array}{c}0.71 \\
(0.40,1.27)\end{array}$ & CNB400 \\
\hline
\end{tabular}

In the lower triangle, the odds ratios have been estimated as treatment in higher order versus treatment in lower order from top to bottom, whereas in the upper triangle the direction of the effects is the opposite. Bold values indicate statistical differences

$B R V 50$ brivaracetam $50 \mathrm{mg} /$ day, BRV100 brivaracetam $100 \mathrm{mg} /$ day, BRV200 brivaracetam $200 \mathrm{mg} /$ day, CNB200 cenobamate $200 \mathrm{mg} / \mathrm{day}$, CNB400 cenobamate $400 \mathrm{mg} /$ day, ESL800 eslicarbazepine acetate $800 \mathrm{mg} /$ day, ESL1200 eslicarbazepine acetate $1200 \mathrm{mg} / \mathrm{day}, L C M 200$ lacosamide $200 \mathrm{mg} /$ day, LCM400 lacosamide $400 \mathrm{mg}$ /day PBO placebo, PER4 perampanel $4 \mathrm{mg} /$ day, $P E R 8$ perampanel $8 \mathrm{mg} / \mathrm{day}, P E R 12$ perampanel $12 \mathrm{mg} /$ day

leading to discontinuation were higher with LCM $400 \mathrm{mg}$ / day (OR 2.60, 95\% CI 1.65-4.09), PER $12 \mathrm{mg}$ /day (OR 3.44, 95\% CI 1.66-7.16), and CNB $400 \mathrm{mg} /$ day (OR $4.35,95 \%$ CI 1.37-13.78) than with LCM $200 \mathrm{mg} /$ day. Similarly, PER 8 $\mathrm{mg} /$ day (OR 2.25, 95\% CI 1.19-4.26), PER $12 \mathrm{mg} /$ day (OR 4.32, 95\% CI 2.24-8.36), and CNB $400 \mathrm{mg} /$ day (OR 5.46, 95\% CI 1.60-18.67) were less tolerated than PER $4 \mathrm{mg} /$ day. A higher rate of participants with TEAEs leading to discontinuation was associated with PER $12 \mathrm{mg} /$ day than PER 8 $\mathrm{mg} /$ day (OR 1.92, 95\% CI 1.29-2.86).

According to SUCRA, BRV and LCM had the highest probabilities of being the best-tolerated options among the individual ASMs for both the outcomes of the occurrence of any TEAE and TEAE leading to discontinuation (Table 5 and Appendix II in the SI); across the individual ASMs at the different dosages, LCM $200 \mathrm{mg}$ /day was the option with the highest probability of being the most tolerated in terms of any TEAE occurrence, and PER $4 \mathrm{mg} /$ day and LCM 200 $\mathrm{mg} /$ day were the options with the lowest likelihood of being associated with TEAEs leading to treatment discontinuation (Table e-5 and Appendix III in the SI). Results from the loop-specific approach for tolerability outcomes are shown in Appendix IV in the SI, and there was no statistically significant incoherence in any loop. Confidence in the evidence for the tolerability outcomes is shown in Appendix V in the SI.

Clustered ranking plots of the ASM network for the efficacy and tolerability outcomes for individual ASMs are shown in Figs. 6, 7, 8 and 9; the corresponding plots by daily dosages are shown in Fig. e-3 in the SI.

\section{Discussion}

\subsection{Summary of Main Results}

The analyses of efficacy outcomes considered in the current NMA indicated that adjunctive CNB was associated with a higher responder rate and the greatest likelihood ranking best for seizure freedom in comparison to add-on BRV, ESL, LCM, and PER.

So far, little has changed in the rate of patients who can achieve seizure control despite the development of many ASMs in the past few decades [37], and there is still the need for novel, more effective therapeutic options. Uncontrolled epilepsy is often disabling, with patients experiencing psychological and social dysfunction, reduced educational and employment prospects, impaired quality of life, and risk of premature death [38]. 
Table 4 Network league table for the seizure freedom

\begin{tabular}{|c|c|c|c|c|c|c|c|c|c|c|c|c|}
\hline PBO & $\begin{array}{c}3.49 \\
(1.36,8.91)\end{array}$ & $\begin{array}{c}3.57 \\
(1.41,8.99)\end{array}$ & $\begin{array}{c}2.95 \\
(0.34,25.26)\end{array}$ & $\begin{array}{c}9.12 \\
(2.53,32.88)\end{array}$ & $\begin{array}{c}6.76 \\
(1.71,26.72)\end{array}$ & $\begin{array}{c}2.66 \\
(0.87,8.08)\end{array}$ & $\begin{array}{c}3.10 \\
(1.03,9.30)\end{array}$ & $\begin{array}{c}3.36 \\
(1.16,9.73)\end{array}$ & $\begin{array}{c}3.83 \\
(1.46,10.02)\end{array}$ & $\begin{array}{c}4.73 \\
(1.73,12.92)\end{array}$ & $\begin{array}{c}10.61 \\
(1.33,84.38)\end{array}$ & $\begin{array}{c}17.68 \\
(2.30,135.90)\end{array}$ \\
\hline $\begin{array}{c}0.29 \\
(0.11,0.73)\end{array}$ & ESL800 & $\begin{array}{c}1.02 \\
(0.53,1.97)\end{array}$ & $\begin{array}{c}0.84 \\
(0.08,8.81)\end{array}$ & $\begin{array}{c}2.62 \\
(0.53,12.81)\end{array}$ & $\begin{array}{c}1.94 \\
(0.37,10.24)\end{array}$ & $\begin{array}{c}0.76 \\
(0.18,3.26)\end{array}$ & $\begin{array}{c}0.89 \\
(0.21,3.77)\end{array}$ & $\begin{array}{c}0.96 \\
(0.23,3.98)\end{array}$ & $\begin{array}{c}1.10 \\
(0.29,4.21)\end{array}$ & $\begin{array}{c}1.36 \\
(0.34,5.36)\end{array}$ & $\begin{array}{c}3.04 \\
(0.31,29.63)\end{array}$ & $\begin{array}{c}5.07 \\
(0.54,47.88)\end{array}$ \\
\hline $\begin{array}{c}0.28 \\
(0.11,0.71)\end{array}$ & $\begin{array}{c}0.98 \\
(0.51,1.88)\end{array}$ & ESL1200 & $\begin{array}{c}0.83 \\
(0.08,8.57)\end{array}$ & $\begin{array}{c}2.56 \\
(0.53,12.43)\end{array}$ & $\begin{array}{c}1.90 \\
(0.36,9.94)\end{array}$ & $\begin{array}{c}0.75 \\
(0.18,3.16)\end{array}$ & $\begin{array}{c}0.87 \\
(0.21,3.65)\end{array}$ & $\begin{array}{c}0.94 \\
(0.23,3.86)\end{array}$ & $\begin{array}{c}1.07 \\
(0.28,4.08)\end{array}$ & $\begin{array}{c}1.33 \\
(0.34,5.20)\end{array}$ & $\begin{array}{c}2.97 \\
(0.31,28.81)\end{array}$ & $\begin{array}{c}4.96 \\
(0.53,46.54)\end{array}$ \\
\hline $\begin{array}{c}0.34 \\
(0.04,2.91)\end{array}$ & $\begin{array}{c}1.18 \\
(0.11,12.35)\end{array}$ & $\begin{array}{c}1.21 \\
(0.12,12.57)\end{array}$ & BRV50 & $\begin{array}{c}3.10 \\
(0.35,27.57)\end{array}$ & $\begin{array}{c}2.30 \\
(0.23,22.60)\end{array}$ & $\begin{array}{c}0.90 \\
(0.08,10.14)\end{array}$ & $\begin{array}{c}1.05 \\
(0.09,11.76)\end{array}$ & $\begin{array}{c}1.14 \\
(0.10,12.55)\end{array}$ & $\begin{array}{c}1.30 \\
(0.12,13.69)\end{array}$ & $\begin{array}{c}1.60 \\
(0.15,17.21)\end{array}$ & $\begin{array}{c}3.60 \\
(0.18,71.37)\end{array}$ & $\begin{array}{c}6.00 \\
(0.31,116.18)\end{array}$ \\
\hline $\begin{array}{c}0.11 \\
(0.03,0.40)\end{array}$ & $\begin{array}{c}0.38 \\
(0.08,1.87)\end{array}$ & $\begin{array}{c}0.39 \\
(0.08,1.90)\end{array}$ & $\begin{array}{c}0.32 \\
(0.04,2.88)\end{array}$ & BRV100 & $\begin{array}{c}0.74 \\
(0.32,1.71)\end{array}$ & $\begin{array}{c}0.29 \\
(0.05,1.59)\end{array}$ & $\begin{array}{c}0.34 \\
(0.06,1.84)\end{array}$ & $\begin{array}{c}0.37 \\
(0.07,1.95)\end{array}$ & $\begin{array}{c}0.42 \\
(0.08,2.09)\end{array}$ & $\begin{array}{c}0.52 \\
(0.10,2.65)\end{array}$ & $\begin{array}{c}1.16 \\
(0.10,13.32)\end{array}$ & $\begin{array}{c}1.94 \\
(0.17,21.57)\end{array}$ \\
\hline $\begin{array}{c}0.15 \\
(0.04,0.58)\end{array}$ & $\begin{array}{c}0.52 \\
(0.10,2.72)\end{array}$ & $\begin{array}{c}0.53 \\
(0.10,2.76)\end{array}$ & $\begin{array}{c}0.44 \\
(0.04,4.29)\end{array}$ & $\begin{array}{c}1.35 \\
(0.58,3.11)\end{array}$ & BRV200 & $\begin{array}{c}0.39 \\
(0.07,2.30)\end{array}$ & $\begin{array}{c}0.46 \\
(0.08,2.66)\end{array}$ & $\begin{array}{c}0.50 \\
(0.09,2.83)\end{array}$ & $\begin{array}{c}0.57 \\
(0.11,3.03)\end{array}$ & $\begin{array}{c}0.70 \\
(0.13,3.84)\end{array}$ & $\begin{array}{c}1.57 \\
(0.13,18.88)\end{array}$ & $\begin{array}{c}2.62 \\
(0.22,30.59)\end{array}$ \\
\hline $\begin{array}{c}0.38 \\
(0.12,1.14)\end{array}$ & $\begin{array}{c}1.31 \\
(0.31,5.62)\end{array}$ & $\begin{array}{c}1.34 \\
(0.32,5.70)\end{array}$ & $\begin{array}{c}1.11 \\
(0.10,12.46)\end{array}$ & $\begin{array}{c}3.43 \\
(0.63,18.73)\end{array}$ & $\begin{array}{c}2.54 \\
(0.43,14.90)\end{array}$ & LCM200 & $\begin{array}{c}1.17 \\
(0.56,2.42)\end{array}$ & $\begin{array}{c}1.26 \\
(0.27,5.89)\end{array}$ & $\begin{array}{c}1.44 \\
(0.33,6.27)\end{array}$ & $\begin{array}{c}1.78 \\
(0.40,7.96)\end{array}$ & $\begin{array}{c}3.99 \\
(0.38,41.98)\end{array}$ & $\begin{array}{c}6.65 \\
(0.65,67.89)\end{array}$ \\
\hline $\begin{array}{c}0.32 \\
(0.11,0.97)\end{array}$ & $\begin{array}{c}1.13 \\
(0.27,4.77)\end{array}$ & $\begin{array}{c}1.15 \\
(0.27,4.84)\end{array}$ & $\begin{array}{c}0.95 \\
(0.09,10.62)\end{array}$ & $\begin{array}{c}2.94 \\
(0.54,15.93)\end{array}$ & $\begin{array}{c}2.18 \\
(0.38,12.68)\end{array}$ & $\begin{array}{c}0.86 \\
(0.41,1.78)\end{array}$ & LCM400 & $\begin{array}{c}1.08 \\
(0.24,5.01)\end{array}$ & $\begin{array}{c}1.24 \\
(0.29,5.32)\end{array}$ & $\begin{array}{c}1.53 \\
(0.34,6.77)\end{array}$ & $\begin{array}{c}3.42 \\
(0.33,35.79)\end{array}$ & $\begin{array}{c}5.71 \\
(0.56,57.88)\end{array}$ \\
\hline $\begin{array}{c}0.30 \\
(0.10,0.86)\end{array}$ & $\begin{array}{c}1.04 \\
(0.25,4.28)\end{array}$ & $\begin{array}{c}1.06 \\
(0.26,4.34)\end{array}$ & $\begin{array}{c}0.88 \\
(0.08,9.64)\end{array}$ & $\begin{array}{c}2.71 \\
(0.51,14.36)\end{array}$ & $\begin{array}{c}2.01 \\
(0.35,11.44)\end{array}$ & $\begin{array}{c}0.79 \\
(0.17,3.68)\end{array}$ & $\begin{array}{c}0.92 \\
(0.20,4.25)\end{array}$ & PER4 & $\begin{array}{c}1.14 \\
(0.53,2.44)\end{array}$ & $\begin{array}{c}1.41 \\
(0.58,3.39)\end{array}$ & $\begin{array}{c}3.16 \\
(0.31,32.46)\end{array}$ & $\begin{array}{c}5.26 \\
(0.53,52.48)\end{array}$ \\
\hline $\begin{array}{c}0.26 \\
(0.10,0.68)\end{array}$ & $\begin{array}{c}0.91 \\
(0.24,3.49)\end{array}$ & $\begin{array}{c}0.93 \\
(0.25,3.54)\end{array}$ & $\begin{array}{c}0.77 \\
(0.07,8.11)\end{array}$ & $\begin{array}{c}2.38 \\
(0.48,11.85)\end{array}$ & $\begin{array}{c}1.77 \\
(0.33,9.46)\end{array}$ & $\begin{array}{c}0.69 \\
(0.16,3.02)\end{array}$ & $\begin{array}{c}0.81 \\
(0.19,3.49)\end{array}$ & $\begin{array}{c}0.88 \\
(0.41,1.88)\end{array}$ & PER8 & $\begin{array}{c}1.23 \\
(0.61,2.51)\end{array}$ & $\begin{array}{c}2.77 \\
(0.28,27.27)\end{array}$ & $\begin{array}{c}4.62 \\
(0.48,44.07)\end{array}$ \\
\hline $\begin{array}{c}0.21 \\
(0.08,0.58)\end{array}$ & $\begin{array}{c}0.74 \\
(0.19,2.92)\end{array}$ & $\begin{array}{c}0.75 \\
(0.19,2.96)\end{array}$ & $\begin{array}{c}0.62 \\
(0.06,6.69)\end{array}$ & $\begin{array}{c}1.93 \\
(0.38,9.85)\end{array}$ & $\begin{array}{c}1.43 \\
(0.26,7.86)\end{array}$ & $\begin{array}{c}0.56 \\
(0.13,2.52)\end{array}$ & $\begin{array}{c}0.66 \\
(0.15,2.91)\end{array}$ & $\begin{array}{c}0.71 \\
(0.30,1.71)\end{array}$ & $\begin{array}{c}0.81 \\
(0.40,1.64)\end{array}$ & PER12 & $\begin{array}{c}2.24 \\
(0.22,22.50)\end{array}$ & $\begin{array}{c}3.74 \\
(0.39,36.36)\end{array}$ \\
\hline $\begin{array}{c}0.09 \\
(0.01,0.75)\end{array}$ & $\begin{array}{c}0.33 \\
(0.03,3.20)\end{array}$ & $\begin{array}{c}0.34 \\
(0.03,3.26)\end{array}$ & $\begin{array}{c}0.28 \\
(0.01,5.50)\end{array}$ & $\begin{array}{c}0.86 \\
(0.08,9.85)\end{array}$ & $\begin{array}{c}0.64 \\
(0.05,7.67)\end{array}$ & $\begin{array}{c}0.25 \\
(0.02,2.64)\end{array}$ & $\begin{array}{c}0.29 \\
(0.03,3.05)\end{array}$ & $\begin{array}{c}0.32 \\
(0.03,3.26)\end{array}$ & $\begin{array}{c}0.36 \\
(0.04,3.55)\end{array}$ & $\begin{array}{c}0.45 \\
(0.04,4.47)\end{array}$ & CNB200 & $\begin{array}{c}1.67 \\
(0.72,3.86)\end{array}$ \\
\hline $\begin{array}{c}0.06 \\
(0.01,0.43)\end{array}$ & $\begin{array}{c}0.20 \\
(0.02,1.86)\end{array}$ & $\begin{array}{c}0.20 \\
(0.02,1.89)\end{array}$ & $\begin{array}{c}0.17 \\
(0.01,3.22)\end{array}$ & $\begin{array}{c}0.52 \\
(0.05,5.74)\end{array}$ & $\begin{array}{c}0.38 \\
(0.03,4.47)\end{array}$ & $\begin{array}{c}0.15 \\
(0.01,1.53)\end{array}$ & $\begin{array}{c}0.18 \\
(0.02,1.78)\end{array}$ & $\begin{array}{c}0.19 \\
(0.02,1.90)\end{array}$ & $\begin{array}{c}0.22 \\
(0.02,2.06)\end{array}$ & $\begin{array}{c}0.27 \\
(0.03,2.60)\end{array}$ & $\begin{array}{c}0.60 \\
(0.26,1.39)\end{array}$ & CNB400 \\
\hline
\end{tabular}

In the lower triangle, the odds ratios have been estimated as treatment in higher order versus treatment in lower order from top to bottom, whereas in the upper triangle the direction of the effects is the opposite. Bold values indicate statistical differences.

$B R V 50$ brivaracetam $50 \mathrm{mg} /$ day, BRV100 brivaracetam $100 \mathrm{mg} /$ day, $B R V 200$ brivaracetam $200 \mathrm{mg} / \mathrm{day}$, CNB200 cenobamate $200 \mathrm{mg} / \mathrm{day}$, CNB400 cenobamate $400 \mathrm{mg} /$ day, ESL800 eslicarbazepine acetate $800 \mathrm{mg} /$ day, ESL1200 eslicarbazepine acetate $1200 \mathrm{mg} / \mathrm{day}, L C M 200$ lacosamide $200 \mathrm{mg} /$ day, LCM400 lacosamide $400 \mathrm{mg}$ /day PBO placebo, PER4 perampanel $4 \mathrm{mg} /$ day, PER 8 perampanel $8 \mathrm{mg} / \mathrm{day}, P E R 12$ perampanel $12 \mathrm{mg} /$ day

Cenobamate has recently been approved for the treatment of focal onset seizures in adults and is characterised by a so-far unique dual complementary mechanism of action. It decreases excitatory currents by inhibiting the persistent component of the sodium current and enhancing the inactivated state of voltage-gated sodium channels [39]. In addition, it enhances inhibitory currents through $\mathrm{GABA}_{\mathrm{A}}$ receptor allosteric modulation and experimental evidence suggests it may target both synaptic and extra-synaptic $\mathrm{GABA}_{\mathrm{A}}$ receptors [39-41]. The comparative analysis of preclinical antiseizure profiles of CNB and other ASMs targeting similar molecular targets suggests that CNB is a mechanistically novel drug [42]. The dual pharmacodynamic activities of CNB on targets regulating both excitatory and inhibitory neurotransmission may contribute to explain its broad preclinical activity and support its clinical efficacy in patients with focal epilepsy [42], although the drug may possess other yet unknown mechanisms of action. The rates of seizure response and seizure freedom observed with CNB compare very favourably with those of the other adjunctive ASMs and bring promise for patients who have difficult-to-control seizures, mainly if the higher dosage can be tolerated. Real-life data overcoming the constraints of the RCT design will complement and further clarify the true therapeutic potentialities and clinical relevance of this newest ASM.
The analyses of tolerability outcomes indicated the good profile of BRV and LCM over the other comparators. Brivaracetam and LCM were associated with a lower proportion of participants experiencing TEAEs in comparison to ESL, and patients treated with PER were associated with a higher risk of experiencing at least one TEAE than BRV. Overall, BRV and LCM had the highest probabilities of being the best-tolerated options for both the outcomes of the occurrence of any TEAE and TEAEs leading to discontinuation.

Brivaracetam is a rationally developed compound characterised by high-affinity binding to synaptic

vesicle protein 2A [43]. Lacosamide, the R-enantiomer of 2-acetamido-N-benzyl-3-methoxypropionamide, selectively enhances the slow inactivation of voltage-gated sodium channels [44]. Both BRV and LCM do not interact with most drug-metabolising enzymes and drug transporters and are associated with few clinically relevant drug-drug interactions $[44,45]$.

The good tolerability profile of BRV versus the other third-generation ASMs has been already demonstrated in prior indirect comparisons [46, 47]. In this regard, it is interesting that more than three-quarters of participants assigned to BRV treatment in the RCTs were taking sodium channel blockers (SCBs) as concomitant ASMs, and the risk of TEAEs in patients treated with BRV within the context of 
Table 5 Ranking according to SUCRA and mean rank for the efficacy and tolerability outcomes

\begin{tabular}{lcc}
\hline Treatment & SUCRA & Mean rank \\
\hline Seizure response & & \\
Brivaracetam & 46.2 & 3.7 \\
Cenobamate & 99.0 & 1.1 \\
Eslicarbazepine acetate & 53.4 & 3.3 \\
Lacosamide & 60.8 & 3.0 \\
Perampanel & 40.7 & 4.0 \\
Placebo & 0.0 & 6.0 \\
Seizure freedom & & \\
Brivaracetam & 72.4 & 2.4 \\
Cenobamate & 88.8 & 1.6 \\
Eslicarbazepine acetate & 47.2 & 3.6 \\
Lacosamide & 37.8 & 4.1 \\
Perampanel & 53.0 & 3.4 \\
Placebo & 0.8 & 6.0 \\
At least one treatment-emergent adverse event & & \\
Brivaracetam & 67.0 & 2.6 \\
Cenobamate & 21.5 & 4.9 \\
Eslicarbazepine acetate & 12.8 & 5.4 \\
Lacosamide & 70.2 & 2.5 \\
Perampanel & 29.6 & 4.5 \\
Placebo & 98.8 & 1.1 \\
At least one treatment-emergent adverse event leading to discon- \\
tinuation & & \\
Brivaracetam & 62.3 & 2.9 \\
Cenobamate & 11.9 & 5.4 \\
Eslicarbazepine acetate & 24.8 & 4.8 \\
Lacosamide & 56.8 & 3.2 \\
Perampanel & 44.5 & 3.8 \\
Placebo & 99.7 & 1.0 \\
\hline
\end{tabular}

SUCRA surface under the cumulative ranking curve

Higher SUCRA values correspond to higher probabilities of better efficacy/tolerability

real-world clinical practice is significantly reduced by the concomitant use of an ASM acting as an SCB [48]. Although to date, there are no experimental data about any advantageous combinations of BRV with other ASMs, additive or synergic pharmacodynamic interactions between BRV and one or more compounds acting on voltage-gated sodium channels may exist and need to be specifically addressed. Further, the ranking of treatments according to tolerability outcomes suggested that the occurrence of any TEAE and the risk of TEAEs leading to discontinuation associated with BRV could not be dose dependent, while the incidence of TEAEs was higher with the increasing doses of CNB, ESL, LCM, and PER.

Both PER and BRV are among the ASMs traditionally associated with the strongest risk of behavioural adverse

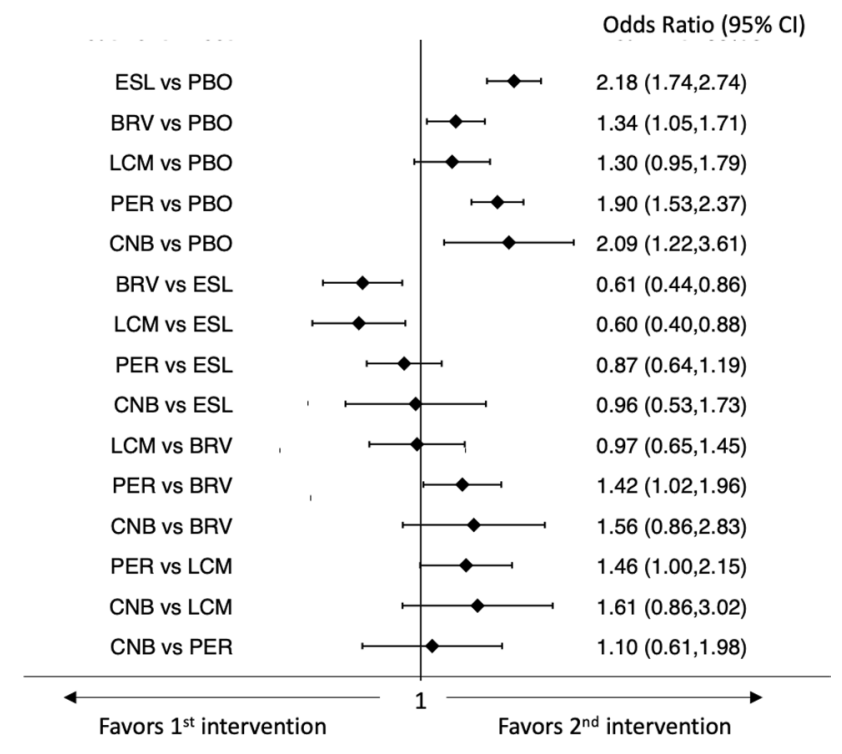

Fig. 4 Interval plot for the tolerability outcome: at least one treatment-emergent adverse event. $B R V$ brivaracetam, $C N B$ cenobamate, $C I$ confidence interval, ESL eslicarbazepine acetate, LCM lacosamide, $P B O$ placebo, $P E R$ perampanel

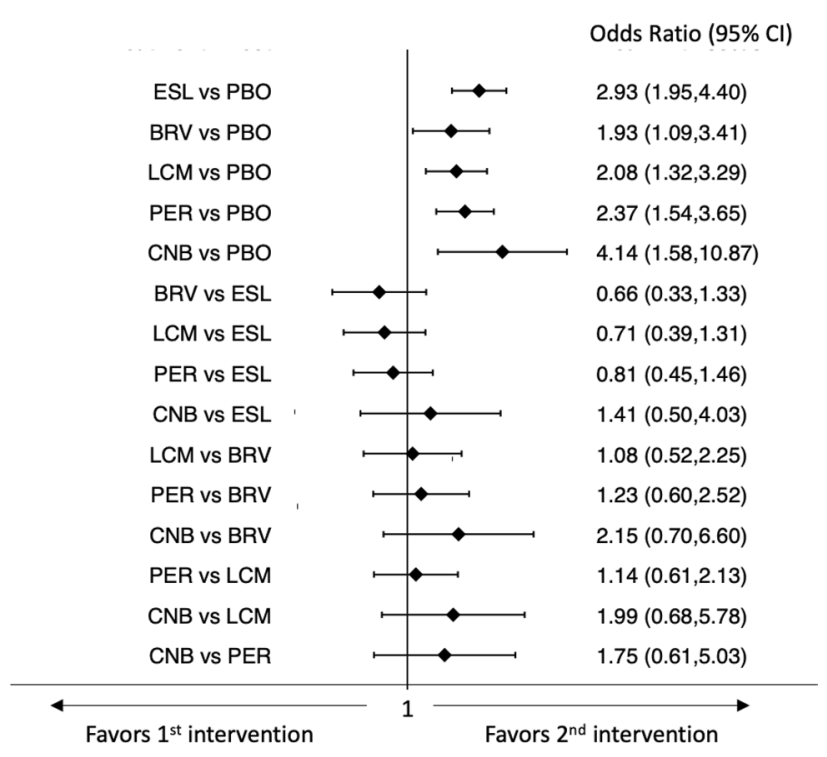

Fig. 5 Interval plot for the tolerability outcome: at least one treatment-emergent adverse event leading to discontinuation. $B R V$ brivaracetam, $C N B$ cenobamate, $C I$ confidence interval, ESL eslicarbazepine acetate, $L C M$ lacosamide, $P B O$ placebo, $P E R$ perampanel

events. Although the incidence of these side effects is higher with PER than with BRV [49], the lack of analyses by individual TEAEs did not allow us to state whether the difference in the tolerability profile was driven by the risk of behavioural adverse events. 
Table 6 Network league table for the occurrence of at least one treatment-emergent adverse event

\begin{tabular}{|c|c|c|c|c|c|c|c|c|c|c|c|c|}
\hline PBO & $\begin{array}{c}1.83 \\
(1.41,2.38)\end{array}$ & $\begin{array}{c}2.62 \\
(2.00,3.43)\end{array}$ & $\begin{array}{c}1.19 \\
(0.79,1.78)\end{array}$ & $\begin{array}{c}1.43 \\
(1.06,1.94)\end{array}$ & $\begin{array}{c}1.36 \\
(0.96,1.92)\end{array}$ & $\begin{array}{c}1.01 \\
(0.71,1.45)\end{array}$ & $\begin{array}{c}1.71 \\
(1.16,2.51)\end{array}$ & $\begin{array}{c}1.32 \\
(0.98,1.77)\end{array}$ & $\begin{array}{c}1.88 \\
(1.44,2.44)\end{array}$ & $\begin{array}{c}3.04 \\
(2.15,4.30)\end{array}$ & $\begin{array}{c}1.36 \\
(0.74,2.49)\end{array}$ & $\begin{array}{c}3.83 \\
(1.81,8.08)\end{array}$ \\
\hline $\begin{array}{c}0.55 \\
(0.42,0.71)\end{array}$ & ESL800 & $\begin{array}{c}1.43 \\
(1.09,1.88)\end{array}$ & $\begin{array}{c}0.65 \\
(0.40,1.05)\end{array}$ & $\begin{array}{c}0.78 \\
(0.53,1.17)\end{array}$ & $\begin{array}{c}0.74 \\
(0.48,1.15)\end{array}$ & $\begin{array}{c}0.55 \\
(0.35,0.86)\end{array}$ & $\begin{array}{c}0.93 \\
(0.59,1.49)\end{array}$ & $\begin{array}{c}0.72 \\
(0.49,1.07)\end{array}$ & $\begin{array}{c}1.02 \\
(0.71,1.48)\end{array}$ & $\begin{array}{c}1.66 \\
(1.08,2.56)\end{array}$ & $\begin{array}{c}0.74 \\
(0.39,1.43)\end{array}$ & $\begin{array}{c}2.09 \\
(0.95,4.61)\end{array}$ \\
\hline $\begin{array}{c}0.38 \\
(0.29,0.50)\end{array}$ & $\begin{array}{c}0.70 \\
(0.53,0.92)\end{array}$ & ESL1200 & $\begin{array}{c}0.45 \\
(0.28,0.74)\end{array}$ & $\begin{array}{c}0.55 \\
(0.37,0.82)\end{array}$ & $\begin{array}{c}0.52 \\
(0.33,0.80)\end{array}$ & $\begin{array}{c}0.39 \\
(0.25,0.61)\end{array}$ & $\begin{array}{c}0.65 \\
(0.41,1.04)\end{array}$ & $\begin{array}{c}0.50 \\
(0.34,0.75)\end{array}$ & $\begin{array}{c}0.72 \\
(0.49,1.04)\end{array}$ & $\begin{array}{c}1.16 \\
(0.75,1.80)\end{array}$ & $\begin{array}{c}0.52 \\
(0.27,1.01)\end{array}$ & $\begin{array}{c}1.46 \\
(0.66,3.24)\end{array}$ \\
\hline $\begin{array}{c}0.84 \\
(0.56,1.27)\end{array}$ & $\begin{array}{c}1.54 \\
(0.95,2.50)\end{array}$ & $\begin{array}{c}2.21 \\
(1.35,3.60)\end{array}$ & BRV50 & $\begin{array}{c}1.21 \\
(0.77,1.89)\end{array}$ & $\begin{array}{c}1.14 \\
(0.69,1.91)\end{array}$ & $\begin{array}{c}0.86 \\
(0.50,1.47)\end{array}$ & $\begin{array}{c}1.44 \\
(0.82,2.52)\end{array}$ & $\begin{array}{c}1.11 \\
(0.67,1.84)\end{array}$ & $\begin{array}{c}1.58 \\
(0.97,2.57)\end{array}$ & $\begin{array}{c}2.56 \\
(1.50,4.38)\end{array}$ & $\begin{array}{c}1.15 \\
(0.55,2.38)\end{array}$ & $\begin{array}{c}3.23 \\
(1.38,7.56)\end{array}$ \\
\hline $\begin{array}{c}0.70 \\
(0.52,0.94)\end{array}$ & $\begin{array}{c}1.28 \\
(0.86,1.90)\end{array}$ & $\begin{array}{c}1.83 \\
(1.22,2.73)\end{array}$ & $\begin{array}{c}0.83 \\
(0.53,1.29)\end{array}$ & BRV100 & $\begin{array}{c}0.95 \\
(0.66,1.35)\end{array}$ & $\begin{array}{c}0.71 \\
(0.44,1.13)\end{array}$ & $\begin{array}{c}1.19 \\
(0.73,1.94)\end{array}$ & $\begin{array}{c}0.92 \\
(0.60,1.40)\end{array}$ & $\begin{array}{c}1.31 \\
(0.88,1.95)\end{array}$ & $\begin{array}{c}2.12 \\
(1.34,3.35)\end{array}$ & $\begin{array}{c}0.95 \\
(0.48,1.86)\end{array}$ & $\begin{array}{c}2.67 \\
(1.19,5.97)\end{array}$ \\
\hline $\begin{array}{c}0.74 \\
(0.52,1.04)\end{array}$ & $\begin{array}{c}1.35 \\
(0.87,2.09)\end{array}$ & $\begin{array}{c}1.93 \\
(1.24,3.00)\end{array}$ & $\begin{array}{c}0.87 \\
(0.52,1.46)\end{array}$ & $\begin{array}{c}1.06 \\
(0.74,1.51)\end{array}$ & BRV200 & $\begin{array}{c}0.75 \\
(0.45,1.23)\end{array}$ & $\begin{array}{c}1.26 \\
(0.75,2.12)\end{array}$ & $\begin{array}{c}0.97 \\
(0.62,1.53)\end{array}$ & $\begin{array}{c}1.38 \\
(0.89,2.14)\end{array}$ & $\begin{array}{c}2.24 \\
(1.37,3.66)\end{array}$ & $\begin{array}{c}1.00 \\
(0.50,2.01)\end{array}$ & $\begin{array}{c}2.82 \\
(1.24,6.44)\end{array}$ \\
\hline $\begin{array}{c}0.99 \\
(0.69,1.41)\end{array}$ & $\begin{array}{c}1.81 \\
(1.16,2.82)\end{array}$ & $\begin{array}{c}2.58 \\
(1.65,4.05)\end{array}$ & $\begin{array}{c}1.17 \\
(0.68,2.01)\end{array}$ & $\begin{array}{c}1.41 \\
(0.88,2.26)\end{array}$ & $\begin{array}{c}1.34 \\
(0.81,2.21)\end{array}$ & LCM200 & $\begin{array}{c}1.68 \\
(1.15,2.48)\end{array}$ & $\begin{array}{c}1.30 \\
(0.82,2.07)\end{array}$ & $\begin{array}{c}1.85 \\
(1.18,2.89)\end{array}$ & $\begin{array}{c}3.00 \\
(1.82,4.94)\end{array}$ & $\begin{array}{c}1.34 \\
(0.66,2.71)\end{array}$ & $\begin{array}{c}3.77 \\
(\mathbf{1 . 6 5 , 8 . 6 5 )}\end{array}$ \\
\hline $\begin{array}{c}0.59 \\
(0.40,0.86)\end{array}$ & $\begin{array}{c}1.07 \\
(0.67,1.71)\end{array}$ & $\begin{array}{c}1.53 \\
(0.96,2.45)\end{array}$ & $\begin{array}{c}0.69 \\
(0.40,1.21)\end{array}$ & $\begin{array}{c}0.84 \\
(0.52,1.37)\end{array}$ & $\begin{array}{c}0.79 \\
(0.47,1.33)\end{array}$ & $\begin{array}{c}0.59 \\
(0.40,0.87)\end{array}$ & LCM400 & $\begin{array}{c}0.77 \\
(0.48,1.25)\end{array}$ & $\begin{array}{c}1.10 \\
(0.69,1.75)\end{array}$ & $\begin{array}{c}1.78 \\
(1.06,2.99)\end{array}$ & $\begin{array}{c}0.80 \\
(0.39,1.63)\end{array}$ & $\begin{array}{c}2.24 \\
(0.97,5.19)\end{array}$ \\
\hline $\begin{array}{c}0.76 \\
(0.56,1.02)\end{array}$ & $\begin{array}{c}1.39 \\
(0.94,2.06)\end{array}$ & $\begin{array}{c}1.98 \\
(1.33,2.96)\end{array}$ & $\begin{array}{c}0.90 \\
(0.54,1.49)\end{array}$ & $\begin{array}{c}1.09 \\
(0.71,1.66)\end{array}$ & $\begin{array}{c}1.03 \\
(0.65,1.62)\end{array}$ & $\begin{array}{c}0.77 \\
(0.48,1.22)\end{array}$ & $\begin{array}{c}1.30 \\
(0.80,2.10)\end{array}$ & PER4 & $\begin{array}{c}1.42 \\
(1.04,1.93)\end{array}$ & $\begin{array}{c}2.30 \\
(1.55,3.43)\end{array}$ & $\begin{array}{c}1.03 \\
(0.53,2.02)\end{array}$ & $\begin{array}{c}2.90 \\
(1.30,6.48)\end{array}$ \\
\hline $\begin{array}{c}0.53 \\
(0.41,0.69)\end{array}$ & $\begin{array}{c}0.98 \\
(0.67,1.41)\end{array}$ & $\begin{array}{c}1.40 \\
(0.96,2.03)\end{array}$ & $\begin{array}{c}0.63 \\
(0.39,1.03)\end{array}$ & $\begin{array}{c}0.76 \\
(0.51,1.14)\end{array}$ & $\begin{array}{c}0.72 \\
(0.47,1.12)\end{array}$ & $\begin{array}{c}0.54 \\
(0.35,0.85)\end{array}$ & $\begin{array}{c}0.91 \\
(0.57,1.45)\end{array}$ & $\begin{array}{c}0.70 \\
(0.52,0.96)\end{array}$ & PER8 & $\begin{array}{c}1.62 \\
(1.13,2.33)\end{array}$ & $\begin{array}{c}0.73 \\
(0.38,1.40)\end{array}$ & $\begin{array}{c}2.04 \\
(0.92,4.51)\end{array}$ \\
\hline $\begin{array}{c}0.33 \\
(0.23,0.47)\end{array}$ & $\begin{array}{c}0.60 \\
(0.39,0.93)\end{array}$ & $\begin{array}{c}0.86 \\
(0.55,1.34)\end{array}$ & $\begin{array}{c}0.39 \\
(0.23,0.67)\end{array}$ & $\begin{array}{c}0.47 \\
(0.30,0.75)\end{array}$ & $\begin{array}{c}0.45 \\
(0.27,0.73)\end{array}$ & $\begin{array}{c}0.33 \\
(0.20,0.55)\end{array}$ & $\begin{array}{c}0.56 \\
(0.33,0.94)\end{array}$ & $\begin{array}{c}0.43 \\
(0.29,0.65)\end{array}$ & $\begin{array}{c}0.62 \\
(0.43,0.89)\end{array}$ & PER12 & $\begin{array}{c}0.45 \\
(0.22,0.90)\end{array}$ & $\begin{array}{c}1.26 \\
(0.55,2.87)\end{array}$ \\
\hline $\begin{array}{c}0.74 \\
(0.40,1.34)\end{array}$ & $\begin{array}{c}1.35 \\
(0.70,2.60)\end{array}$ & $\begin{array}{c}1.92 \\
(0.99,3.73)\end{array}$ & $\begin{array}{c}0.87 \\
(0.42,1.80)\end{array}$ & $\begin{array}{c}1.05 \\
(0.54,2.07)\end{array}$ & $\begin{array}{c}1.00 \\
(0.50,2.00)\end{array}$ & $\begin{array}{c}0.75 \\
(0.37,1.51)\end{array}$ & $\begin{array}{c}1.26 \\
(0.61,2.57)\end{array}$ & $\begin{array}{c}0.97 \\
(0.50,1.90)\end{array}$ & $\begin{array}{c}1.38 \\
(0.71,2.66)\end{array}$ & $\begin{array}{c}2.23 \\
(1.11,4.48)\end{array}$ & CNB200 & $\begin{array}{c}2.81 \\
(1.31,6.03)\end{array}$ \\
\hline $\begin{array}{c}0.26 \\
(0.12,0.55)\end{array}$ & $\begin{array}{c}0.48 \\
(0.22,1.06)\end{array}$ & $\begin{array}{c}0.68 \\
(0.31,1.51)\end{array}$ & $\begin{array}{c}0.31 \\
(0.13,0.73)\end{array}$ & $\begin{array}{c}0.37 \\
(0.17,0.84)\end{array}$ & $\begin{array}{c}0.35 \\
(0.16,0.81)\end{array}$ & $\begin{array}{c}0.26 \\
(0.12,0.61)\end{array}$ & $\begin{array}{c}0.45 \\
(0.19,1.03)\end{array}$ & $\begin{array}{c}0.34 \\
(0.15,0.77)\end{array}$ & $\begin{array}{c}0.49 \\
(0.22,1.08)\end{array}$ & $\begin{array}{c}0.79 \\
(0.35,1.81)\end{array}$ & $\begin{array}{c}0.36 \\
(0.17,0.76)\end{array}$ & CNB400 \\
\hline
\end{tabular}

In the lower triangle, the odds ratios have been estimated as treatment in higher order versus treatment in lower order from top to bottom, whereas in the upper triangle the direction of the effects is the opposite. Bold values indicate statistical differences.

$B R V 50$ brivaracetam $50 \mathrm{mg} /$ day, BRV100 brivaracetam $100 \mathrm{mg} /$ day, $B R V 200$ brivaracetam $200 \mathrm{mg} / \mathrm{day}$, CNB200 cenobamate $200 \mathrm{mg} / \mathrm{day}$, CNB400 cenobamate $400 \mathrm{mg} /$ day, ESL800 eslicarbazepine acetate $800 \mathrm{mg} /$ day, ESL1200 eslicarbazepine acetate $1200 \mathrm{mg} / \mathrm{day}, L C M 200$ lacosamide $200 \mathrm{mg} /$ day, LCM400 lacosamide $400 \mathrm{mg}$ /day PBO placebo, PER4 perampanel $4 \mathrm{mg} /$ day, PER 8 perampanel $8 \mathrm{mg} / \mathrm{day}, P E R 12$ perampanel $12 \mathrm{mg} /$ day

Also, LCM was associated with a lower proportion of patients experiencing at least one TEAE compared to ESL. Both LCM and ESL belong to the class of SCBs and reduce the voltage-gated sodium channels availability through enhancement of slow inactivation. However, it is worthy of note that distinctive characteristics exist between the two compounds, including the higher interaction of LCM with fast inactivation gating [50]. The possible contribution of such pharmacodynamic features to dictate differential tolerability in clinical practice has never been investigated and remains an attractive theme to explore.

Finally, CNB ranked as the treatment associated with the highest likelihood of TEAE occurrence, mainly at the highest daily dosage. In this regard, the rapid titration of $100 \mathrm{mg} /$ week from 200 to $400 \mathrm{mg}$, and the impossibility as per study protocol to adjust the dose of concomitant ASMs, might have contributed to the higher rates of TEAEs in the $400 \mathrm{mg}$ group. Interestingly, a slightly higher incidence of TEAEs has been observed among patients treated with CNB and concomitantly taking SCBs compared with those not taking them [51]. Further, as CNB inhibits cytochrome P450 (CYP), 2C19 and can inhibit or induce CYP2B6 and 3A4, pharmacokinetic drug-drug interactions occur; for example, CNB can increase plasma concentrations of desmethylclobazam, the active metabolite of clobazam, and a reduction in clobazam dose should be considered to avoid adverse events [39]. Considering the remarkable efficacy profile of CNB, it is important to be aware of the potential interactions with concomitant ASMs and ensure that patients are able to tolerate the introduction of the drug into the existing therapeutic regimen. Special caution and slow titration or stepwise tapering off should be considered in patients on other SCBs or GABA-ergic drugs to improve tolerability and minimise the risk of treatment failure due to TEAEs.

\subsection{Strengths and Limitations}

This NMA is the first to include CNB, the newest approved drug for treating focal-onset seizures, in comparative analyses of the efficacy and safety of ASMs and it updates the currently available systematic reviews of evidence [46, 47, 52]. Efficacy and safety analyses were performed according to actual licensed maintenance daily doses of ASMs and the estimates of seizure responder rates during the maintenance period can reflect steady-state drug levels at the target dose more accurately than data referring to the entire treatment phase, including the titration. The "pragmatic ITT" approach used for the seizure freedom outcome, which defines the percentage of seizure free completers, represents a more conservative method of measuring seizure freedom and provides 
Table 7 Network league table for the occurrence of at least one treatment-emergent adverse event leading to discontinuation

\begin{tabular}{|c|c|c|c|c|c|c|c|c|c|c|c|c|}
\hline PBO & $\begin{array}{c}1.95 \\
(1.23,3.09)\end{array}$ & $\begin{array}{c}4.10 \\
(2.67,6.30)\end{array}$ & $\begin{array}{c}2.02 \\
(0.78,5.25)\end{array}$ & $\begin{array}{c}2.04 \\
(1.06,3.94)\end{array}$ & $\begin{array}{c}1.70 \\
(0.80,3.62)\end{array}$ & $\begin{array}{c}1.17 \\
(0.67,2.04)\end{array}$ & $\begin{array}{c}3.05 \\
(1.90,4.90)\end{array}$ & $\begin{array}{c}0.93 \\
(0.46,1.88)\end{array}$ & $\begin{array}{c}2.10 \\
(1.30,3.40)\end{array}$ & $\begin{array}{c}4.03 \\
(2.50,6.50)\end{array}$ & $\begin{array}{c}3.25 \\
(1.14,9.29)\end{array}$ & $\begin{array}{c}5.09 \\
(1.85,14.00)\end{array}$ \\
\hline $\begin{array}{c}0.51 \\
(0.32,0.81)\end{array}$ & ESL800 & $\begin{array}{c}2.11 \\
(1.49,2.98)\end{array}$ & $\begin{array}{c}1.04 \\
(0.36,2.99)\end{array}$ & $\begin{array}{c}1.05 \\
(0.47,2.34)\end{array}$ & $\begin{array}{c}0.88 \\
(0.36,2.12)\end{array}$ & $\begin{array}{c}0.60 \\
(0.29,1.24)\end{array}$ & $\begin{array}{c}1.57 \\
(0.81,3.03)\end{array}$ & $\begin{array}{c}0.48 \\
(0.21,1.11)\end{array}$ & $\begin{array}{c}1.08 \\
(0.55,2.10)\end{array}$ & $\begin{array}{c}2.07 \\
(1.07,4.02)\end{array}$ & $\begin{array}{c}1.67 \\
(0.53,5.26)\end{array}$ & $\begin{array}{c}2.62 \\
(0.86,7.95)\end{array}$ \\
\hline $\begin{array}{c}0.24 \\
(0.16,0.37)\end{array}$ & $\begin{array}{c}0.47 \\
(0.34,0.67)\end{array}$ & ESL1200 & $\begin{array}{c}0.49 \\
(0.17,1.40)\end{array}$ & $\begin{array}{c}0.50 \\
(0.23,1.09)\end{array}$ & $\begin{array}{c}0.42 \\
(0.17,0.99)\end{array}$ & $\begin{array}{c}0.29 \\
(0.14,0.58)\end{array}$ & $\begin{array}{c}0.74 \\
(0.39,1.41)\end{array}$ & $\begin{array}{c}0.23 \\
(0.10,0.52)\end{array}$ & $\begin{array}{c}0.51 \\
(0.27,0.97)\end{array}$ & $\begin{array}{c}0.98 \\
(0.52,1.87)\end{array}$ & $\begin{array}{c}0.79 \\
(0.26,2.46)\end{array}$ & $\begin{array}{c}1.24 \\
(0.41,3.72)\end{array}$ \\
\hline $\begin{array}{c}0.50 \\
(0.19,1.29)\end{array}$ & $\begin{array}{c}0.96 \\
(0.33,2.79)\end{array}$ & $\begin{array}{c}2.03 \\
(0.71,5.79)\end{array}$ & BRV50 & $\begin{array}{c}1.01 \\
(0.37,2.78)\end{array}$ & $\begin{array}{c}0.84 \\
(0.27,2.60)\end{array}$ & $\begin{array}{c}0.58 \\
(0.19,1.75)\end{array}$ & $\begin{array}{c}1.51 \\
(0.52,4.39)\end{array}$ & $\begin{array}{c}0.46 \\
(0.14,1.51)\end{array}$ & $\begin{array}{c}1.04 \\
(0.36,3.03)\end{array}$ & $\begin{array}{c}2.00 \\
(0.69,5.81)\end{array}$ & $\begin{array}{c}1.61 \\
(0.39,6.66)\end{array}$ & $\begin{array}{c}2.52 \\
(0.63,10.14)\end{array}$ \\
\hline $\begin{array}{c}0.49 \\
(0.25,0.95)\end{array}$ & $\begin{array}{c}0.95 \\
(0.43,2.13)\end{array}$ & $\begin{array}{c}2.01 \\
(0.92,4.42)\end{array}$ & $\begin{array}{c}0.99 \\
(0.36,2.72)\end{array}$ & BRV100 & $\begin{array}{c}0.84 \\
(0.44,1.60)\end{array}$ & $\begin{array}{c}0.57 \\
(0.24,1.36)\end{array}$ & $\begin{array}{c}1.49 \\
(0.66,3.37)\end{array}$ & $\begin{array}{c}0.46 \\
(0.17,1.20)\end{array}$ & $\begin{array}{c}1.03 \\
(0.45,2.33)\end{array}$ & $\begin{array}{c}1.98 \\
(0.88,4.46)\end{array}$ & $\begin{array}{c}1.59 \\
(0.46,5.51)\end{array}$ & $\begin{array}{c}2.50 \\
(0.75,8.35)\end{array}$ \\
\hline $\begin{array}{c}0.59 \\
(0.28,1.25)\end{array}$ & $\begin{array}{c}1.14 \\
(0.47,2.77)\end{array}$ & $\begin{array}{c}2.41 \\
(1.01,5.73)\end{array}$ & $\begin{array}{c}1.18 \\
(0.38,3.65)\end{array}$ & $\begin{array}{c}1.20 \\
(0.62,2.29)\end{array}$ & BRV200 & $\begin{array}{c}0.69 \\
(0.27,1.75)\end{array}$ & $\begin{array}{c}1.79 \\
(0.73,4.36)\end{array}$ & $\begin{array}{c}0.55 \\
(0.20,1.53)\end{array}$ & $\begin{array}{c}1.23 \\
(0.50,3.01)\end{array}$ & $\begin{array}{c}2.37 \\
(0.97,5.78)\end{array}$ & $\begin{array}{c}1.91 \\
(0.52,6.95)\end{array}$ & $\begin{array}{c}2.99 \\
(0.85,10.55)\end{array}$ \\
\hline $\begin{array}{c}0.85 \\
(0.49,1.49)\end{array}$ & $\begin{array}{c}1.66 \\
(0.81,3.42)\end{array}$ & $\begin{array}{c}3.50 \\
(1.74,7.06)\end{array}$ & $\begin{array}{c}1.72 \\
(0.57,5.20)\end{array}$ & $\begin{array}{c}1.74 \\
(0.74,4.12)\end{array}$ & $\begin{array}{c}1.45 \\
(0.57,3.71)\end{array}$ & LCM200 & $\begin{array}{c}2.60 \\
(1.65,4.09)\end{array}$ & $\begin{array}{c}0.80 \\
(0.33,1.94)\end{array}$ & $\begin{array}{c}1.79 \\
(0.86,3.73)\end{array}$ & $\begin{array}{c}3.44 \\
(1.66,7.16)\end{array}$ & $\begin{array}{c}2.78 \\
(0.85,9.10)\end{array}$ & $\begin{array}{c}4.35 \\
(1.37,13.78)\end{array}$ \\
\hline $\begin{array}{c}0.33 \\
(0.20,0.53)\end{array}$ & $\begin{array}{c}0.64 \\
(0.33,1.24)\end{array}$ & $\begin{array}{c}1.35 \\
(0.71,2.55)\end{array}$ & $\begin{array}{c}0.66 \\
(0.23,1.92)\end{array}$ & $\begin{array}{c}0.67 \\
(0.30,1.51)\end{array}$ & $\begin{array}{c}0.56 \\
(0.23,1.36)\end{array}$ & $\begin{array}{c}0.38 \\
(0.24,0.60)\end{array}$ & LCM400 & $\begin{array}{c}0.31 \\
(0.13,0.71)\end{array}$ & $\begin{array}{c}0.69 \\
(0.35,1.35)\end{array}$ & $\begin{array}{c}1.32 \\
(0.68,2.59)\end{array}$ & $\begin{array}{c}1.07 \\
(0.34,3.38)\end{array}$ & $\begin{array}{c}1.67 \\
(0.55,5.10)\end{array}$ \\
\hline $\begin{array}{c}1.07 \\
(0.53,2.16)\end{array}$ & $\begin{array}{c}2.09 \\
(0.90,4.82)\end{array}$ & $\begin{array}{c}4.40 \\
(1.94,9.99)\end{array}$ & $\begin{array}{c}2.16 \\
(0.66,7.07)\end{array}$ & $\begin{array}{c}2.19 \\
(0.84,5.72)\end{array}$ & $\begin{array}{c}1.83 \\
(0.65,5.11)\end{array}$ & $\begin{array}{c}1.26 \\
(0.51,3.07)\end{array}$ & $\begin{array}{c}3.27 \\
(1.40,7.61)\end{array}$ & PER4 & $\begin{array}{c}2.25 \\
(1.19,4.26)\end{array}$ & $\begin{array}{c}4.32 \\
(2.24,8.36)\end{array}$ & $\begin{array}{c}3.49 \\
(0.99,12.31)\end{array}$ & $\begin{array}{c}5.46 \\
(1.60,18.67)\end{array}$ \\
\hline $\begin{array}{c}0.48 \\
(0.29,0.77)\end{array}$ & $\begin{array}{c}0.93 \\
(0.48,1.81)\end{array}$ & $\begin{array}{c}1.96 \\
(1.03,3.73)\end{array}$ & $\begin{array}{c}0.96 \\
(0.33,2.80)\end{array}$ & $\begin{array}{c}0.97 \\
(0.43,2.20)\end{array}$ & $\begin{array}{c}0.81 \\
(0.33,1.99)\end{array}$ & $\begin{array}{c}0.56 \\
(0.27,1.16)\end{array}$ & $\begin{array}{c}1.45 \\
(0.74,2.86)\end{array}$ & $\begin{array}{c}0.44 \\
(0.23,0.84)\end{array}$ & PER8 & $\begin{array}{c}1.92 \\
(1.29,2.86)\end{array}$ & $\begin{array}{c}1.55 \\
(0.49,4.92)\end{array}$ & $\begin{array}{c}2.43 \\
(0.79,7.44)\end{array}$ \\
\hline $\begin{array}{c}0.25 \\
(0.15,0.40)\end{array}$ & $\begin{array}{c}0.48 \\
(0.25,0.94)\end{array}$ & $\begin{array}{c}1.02 \\
(0.54,1.93)\end{array}$ & $\begin{array}{c}0.50 \\
(0.17,1.46)\end{array}$ & $\begin{array}{c}0.51 \\
(0.22,1.14)\end{array}$ & $\begin{array}{c}0.42 \\
(0.17,1.03)\end{array}$ & $\begin{array}{c}0.29 \\
(0.14,0.60)\end{array}$ & $\begin{array}{c}0.76 \\
(0.39,1.48)\end{array}$ & $\begin{array}{c}0.23 \\
(0.12,0.45)\end{array}$ & $\begin{array}{c}0.52 \\
(0.35,0.77)\end{array}$ & PER12 & $\begin{array}{c}0.81 \\
(0.25,2.56)\end{array}$ & $\begin{array}{c}1.26 \\
(0.41,3.86)\end{array}$ \\
\hline $\begin{array}{c}0.31 \\
(0.11,0.88)\end{array}$ & $\begin{array}{c}0.60 \\
(0.19,1.88)\end{array}$ & $\begin{array}{c}1.26 \\
(0.41,3.92)\end{array}$ & $\begin{array}{c}0.62 \\
(0.15,2.57)\end{array}$ & $\begin{array}{c}0.63 \\
(0.18,2.17)\end{array}$ & $\begin{array}{c}0.52 \\
(0.14,1.91)\end{array}$ & $\begin{array}{c}0.36 \\
(0.11,1.18)\end{array}$ & $\begin{array}{c}0.94 \\
(0.30,2.97)\end{array}$ & $\begin{array}{c}0.29 \\
(0.08,1.01)\end{array}$ & $\begin{array}{c}0.65 \\
(0.20,2.05)\end{array}$ & $\begin{array}{c}1.24 \\
(0.39,3.93)\end{array}$ & CNB200 & $\begin{array}{c}1.57 \\
(0.76,3.21)\end{array}$ \\
\hline $\begin{array}{c}0.20 \\
(0.07,0.54)\end{array}$ & $\begin{array}{c}0.38 \\
(0.13,1.16)\end{array}$ & $\begin{array}{c}0.81 \\
(0.27,2.42)\end{array}$ & $\begin{array}{c}0.40 \\
(0.10,1.59)\end{array}$ & $\begin{array}{c}0.40 \\
(0.12,1.34)\end{array}$ & $\begin{array}{c}0.33 \\
(0.09,1.18)\end{array}$ & $\begin{array}{c}0.23 \\
(0.07,0.73)\end{array}$ & $\begin{array}{c}0.60 \\
(0.20,1.83)\end{array}$ & $\begin{array}{c}0.18 \\
(0.05,0.63)\end{array}$ & $\begin{array}{c}0.41 \\
(0.13,1.26)\end{array}$ & $\begin{array}{c}0.79 \\
(0.26,2.42)\end{array}$ & $\begin{array}{c}0.64 \\
(0.31,1.31)\end{array}$ & CNB400 \\
\hline
\end{tabular}

In the lower triangle, the odds ratios have been estimated as treatment in higher order versus treatment in lower order from top to bottom, whereas in the upper triangle the direction of the effects is the opposite. Bold values indicate statistical differences.

BRV50 brivaracetam $50 \mathrm{mg} /$ day, BRV100 brivaracetam $100 \mathrm{mg} /$ day, BRV200 brivaracetam $200 \mathrm{mg} / \mathrm{day}$, CNB200 cenobamate $200 \mathrm{mg} / \mathrm{day}$, CNB400 cenobamate $400 \mathrm{mg} /$ day, ESL800 eslicarbazepine acetate $800 \mathrm{mg} / \mathrm{day}$, ESL1200 eslicarbazepine acetate $1200 \mathrm{mg} / \mathrm{day}, L C M 200$ lacosamide $200 \mathrm{mg} /$ day, LCM400 lacosamide $400 \mathrm{mg} /$ day $P B O$ placebo, $P E R 4$ perampanel $4 \mathrm{mg} /$ day, $P E R 8$ perampanel $8 \mathrm{mg} / \mathrm{day}, P E R 12$ perampanel $12 \mathrm{mg} /$ day

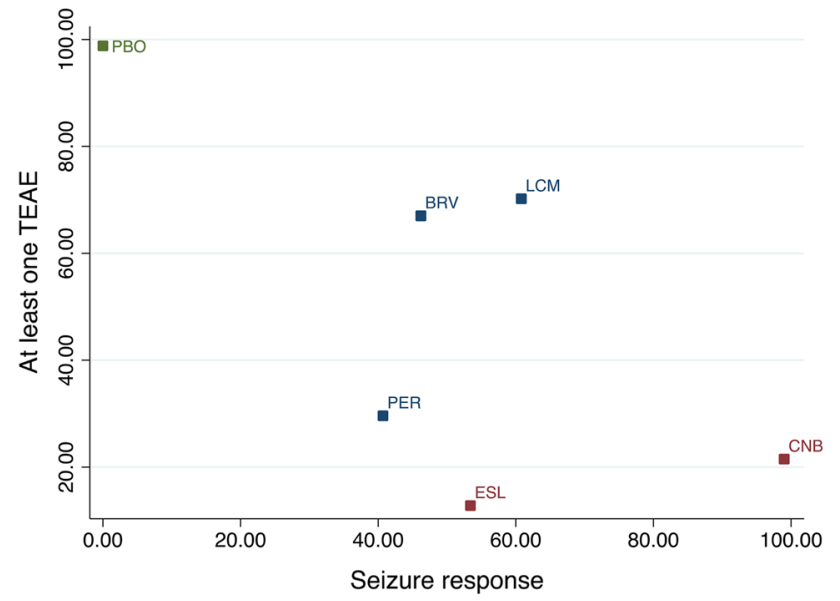

Fig. 6 Clustered ranking plot of the antiseizure medications network for the efficacy and tolerability outcomes: seizure response and occurrence of at least one treatment-emergent adverse event. Ranking plots are based on cluster analysis of SUCRA values for two different outcomes: efficacy and tolerability. Note: $Y$ and $X$ axes represent the cumulative ranking curve (SUCRA) to rank each interventioni.e., probability between 0 to 1 of an intervention being superior in efficacy ( $X$ axis) or tolerability ( $Y$ axis). Higher SUCRA values correspond to higher probabilities of better efficacy/tolerability. Each colour represents a group of treatments that belong to the same cluster. $B R V$ brivaracetam, $C N B$ cenobamate, $C I$ confidence interval, $E S L$ eslicarbazepine acetate, $L C M$ lacosamide, $P B O$ placebo, $P E R$ perampanel, SUCRA surface under the cumulative ranking curve more reliable and informative estimates of treatment efficacy as opposed to "last observation carried forward" methodology, whereby patients who were seizure-free up to dropping out of a study can be classed as being seizure-free [10]. Although the seizure-free numbers in add-on RCTs may not be predictive of seizure freedom in clinical practice, they do represent a reference point when assessing ASMs and an often-discussed marker of how potentially useful an ASM will be in refractory patients [10]. In this regard, the "pragmatic ITT" analysis more closely represents the information on seizure freedom that clinicians seek. Notably, only a few patients achieved seizure freedom in all included studies, and this may have led to a lack of sufficient statistical power to detect a significant difference between the comparators [53].

The validity of indirect comparisons relies upon the similarity and methodological quality of the individual RCTs being indirectly compared [54]. In the present NMA, we used strict inclusion criteria to minimize, as much as possible, any source of heterogeneity; all the included trials were overall clinically and methodologically homogeneous and comparable, and no trial showed a high risk of bias. Although there is no consensus about the optimal length of the treatment period, the inclusion of studies with a maintenance phase of 12 weeks or longer met the guideline on 


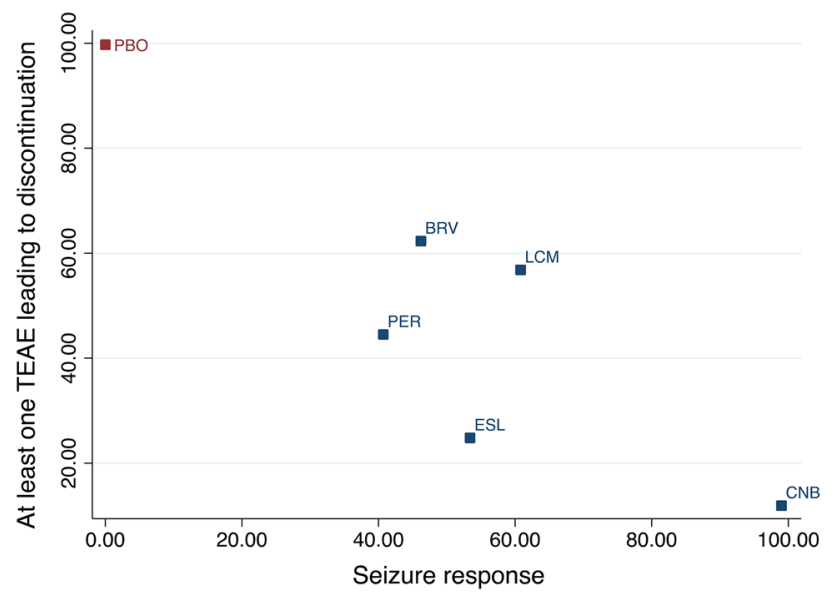

Fig. 7 Clustered ranking plot of the antiseizure medications network for the efficacy and tolerability outcomes: seizure response and occurrence of at least one treatment-emergent adverse event leading to treatment discontinuation. Ranking plots are based on cluster analysis of SUCRA values for two different outcomes: efficacy and tolerability. Note: $Y$ and $\mathrm{X}$ axes represent the cumulative ranking curve (SUCRA) to rank each intervention-i.e., probability between 0 to 1 of an intervention being superior in efficacy ( $X$ axis) or tolerability ( $Y$ axis). Higher SUCRA values correspond to higher probabilities of better efficacy/tolerability. Each colour represents a group of treatments that belong to the same cluster. $B R V$ brivaracetam, $C N B$ cenobamate, $C I$ confidence interval, ESL eslicarbazepine acetate, $L C M$ lacosamide, $P B O$ placebo, $P E R$ perampanel, SUCRA surface under the cumulative ranking curve

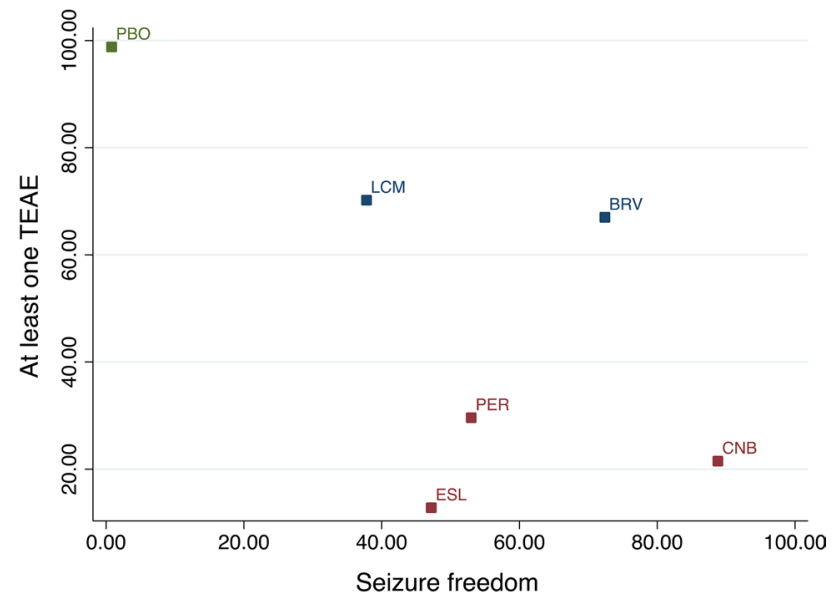

Fig. 8 Clustered ranking plot of the antiseizure medications network for the efficacy and tolerability outcomes: seizure freedom and occurrence of at least one treatment-emergent adverse event. Ranking plots are based on cluster analysis of SUCRA values for two different outcomes: efficacy and tolerability. Note: $Y$ and $X$ axes represent the cumulative ranking curve (SUCRA) to rank each intervention-i.e., probability between 0 to 1 of an intervention being superior in efficacy ( $X$ axis) or tolerability ( $Y$ axis). Higher SUCRA values correspond to higher probabilities of better efficacy/tolerability. Each colour represents a group of treatments that belong to the same cluster. $B R V$ brivaracetam, $C N B$ cenobamate, $C I$ confidence interval, $E S L$ eslicarbazepine acetate, $L C M$ lacosamide, $P B O$ placebo, $P E R$ perampanel, SUCRA surface under the cumulative ranking curve

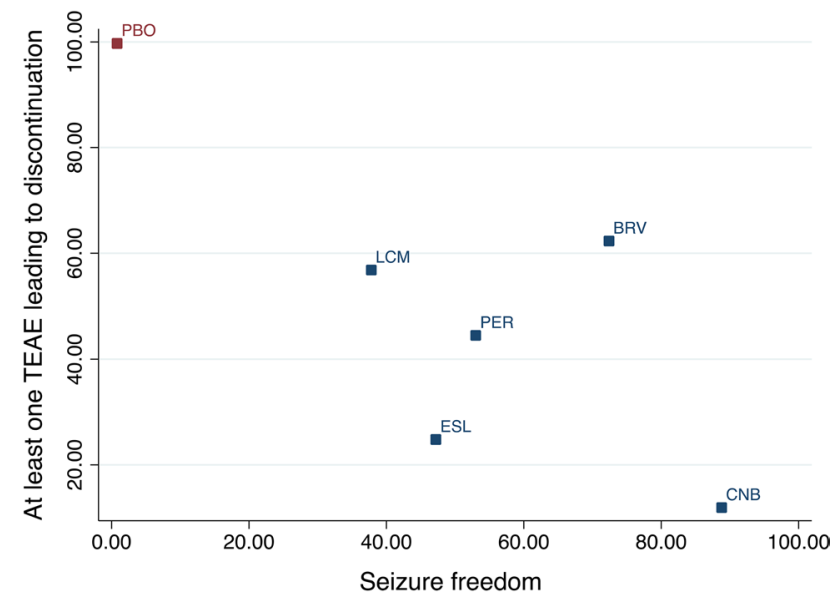

Fig. 9 Clustered ranking plot of the antiseizure medications network for the efficacy and tolerability outcomes: seizure freedom and occurrence of at least one treatment-emergent adverse event leading to treatment discontinuation. Ranking plots are based on cluster analysis of SUCRA values for two different outcomes: efficacy and tolerability. Note: $Y$ and $X$ axes represent the cumulative ranking curve (SUCRA) to rank each intervention-i.e., probability between 0 to 1 of an intervention being superior in efficacy ( $X$ axis) or tolerability ( $Y$ axis). Higher SUCRA values correspond to higher probabilities of better efficacy/tolerability. Each colour represents a group of treatments that belong to the same cluster. $B R V$ brivaracetam, $C N B$ cenobamate, $C I$ confidence interval, ESL eslicarbazepine acetate, $L C M$ lacosamide, $P B O$ placebo, $P E R$ perampanel, SUCRA surface under the cumulative ranking curve

clinical investigation of medicinal products in the treatment of epileptic disorders, which recommends that the maintenance period should last at least 12 weeks to establish that efficacy is not short lasting [55]. However, despite the similarity of the included RCTs, some differences in characteristics of trials and populations such as treatment duration and concomitant medications need to be acknowledged. Studies with shorter double-blind treatment periods may have underestimated the occurrence of TEAEs compared to those with longer time frames available for tolerability assessment, and a higher burden of concomitant ASMs may have resulted in an increased frequency of TEAEs. Further, the lack of efficacy and tolerability data according to the mechanism of action of concomitant ASMs prevents to explore or confirm potential favourable or unfavourable drug interactions and combinations $[48,56]$.

The NMA also inherited the general limitations of the RCTs of adjunctive ASMs, including the short length of observation and the highly selected inclusion and exclusion criteria for enrolment, which do not account for the inherent variability of epilepsy and the need to tailor treatment according to individual characteristics, hence, limiting the external validity of the results. Although the $\geq 50 \%$ reduction in baseline seizure frequency represents one of the outcomes required for licensing studies, it lacks information 
on the actual effect of the treatment on the quality of life of patients. Further, the reliability of seizure count, as documented in clinical trials by self-reported calendar diaries, may be affected by issues like noncompliance with diary maintenance, poor accuracy in reporting, and limited awareness of seizures [57, 58]. Accordingly, studies considering patient-reported outcomes and new approaches for collecting reliable information about seizure control are warranted to fully assess the potential of treatments in the management of patients with epilepsy. In addition, all studies were funded by pharmaceutical companies. Importantly, data about efficacy and safety of CNB are obtained from one single trial and some of the results are associated with wide confidence intervals. In this regard, imprecision and uncertainty associated with low event rates can lead to extreme results and, in turn, influence the rankings of treatments. As ranks are sensitive to imprecision, dose-dependent relationships need to be interpreted with caution and no definitive conclusions can be drawn for trends that do not formally reach statistical significance. Finally, the protocol of this NMA was not registered previously, and the lack of information about economic issues does not allow to address cost-effectiveness analyses and provide further guidance for clinical decisions.

\section{Conclusion}

The comparative analyses of trials' data have shown that CNB ranked best for efficacy outcomes standing out against the comparators, and BRV and LCM had the highest probabilities of being the best-tolerated options among the ASMs considered in this NMA. Ideally, head-to-head trials may allow definite conclusions on the comparative efficacy and tolerability of ASMs. It is unlikely that such RCTs will be performed. Although NMAs cannot replace studies directly comparing two or more drugs, they may offer reliable evidence of the relative efficacy and safety, provide useful information about the hierarchy of competing interventions, and may inform and guide physicians in clinical decision making $[5,59,60]$.

Supplementary Information The online version contains supplementary material available at https://doi.org/10.1007/s40265-021-01661-4.

\section{Declarations}

Funding This study was not funded.

Conflicts of interest Simona Lattanzi has received speaker's or consultancy fees from Angelini Pharma, Eisai, GW Pharmaceuticals, and UCB Pharma and has served on advisory boards for Angelini Pharma, Arvelle Therapeutics, BIAL, and GW Pharmaceuticals. Eugen Trinka reports personal fees from EVER Pharma, Marinus, Angelini, Arvelle Therapeutics, Argenix, Medtronic, Bial-Portela \& $C^{\text {a }}$, NewBridge, GL
Pharma, GlaxoSmithKline, Boehringer Ingelheim, LivaNova, Eisai, Epilog, UCB Pharma, Biogen, Genzyme Sanofi, Takeda, and Actavis; his institution received grants from Biogen, UCB Pharma, Eisai, Red Bull, Merck, Bayer, the European Union, FWF Osterreichischer Fond zur Wissenschaftsforderung, Bundesministerium für Wissenschaft und Forschung, and Jubiläumsfond der Österreichischen Nationalbank outside the submitted work. Gaetano Zaccara has received speaker's or consultancy fees from Eisai, Jazz Pharmaceuticals, and UCB Pharma and has served on advisory board for GW Pharmaceuticals. Pasquale Striano received fees and research grants from GW Pharmaceuticals, Zogenyx, Biomarin, and Kolfarma. Emilio Russo has received speaker fees or funding from, and has participated in advisory boards for Angelini, Arvelle Therapeutics, Eisai, Kolfarma, Pfizer, GW Pharmaceuticals, UCB Pharma, and Lundbeck. Francesco Brigo acted as a consultant for Eisai. Cinzia Del Giovane and Mauro Silvestrini have no conflicts of interest directly relevant to the content of this study.

Ethics approval Not applicable.

Consent to participate Not applicable.

Consent for publication Not applicable

Availability of data and material Not applicable.

Code availability Not applicable

Author contributions SL designed and conceptualised the study, carried out the data analyses, and drafted the manuscript. CDG carried out the data analyses. All authors critically revised the manuscript for important intellectual content. All authors approved the final manuscript for submission and agree to be accountable for all aspects of the work.

Open Access This article is licensed under a Creative Commons Attribution-NonCommercial 4.0 International License, which permits any non-commercial use, sharing, adaptation, distribution and reproduction in any medium or format, as long as you give appropriate credit to the original author(s) and the source, provide a link to the Creative Commons licence, and indicate if changes were made. The images or other third party material in this article are included in the article's Creative Commons licence, unless indicated otherwise in a credit line to the material. If material is not included in the article's Creative Commons licence and your intended use is not permitted by statutory regulation or exceeds the permitted use, you will need to obtain permission directly from the copyright holder. To view a copy of this licence, visit http://creativecommons.org/licenses/by-nc/4.0/.

\section{References}

1. Hirtz D, Thurman DJ, Gwinn-Hardy K, Mohamed M, Chaudhuri AR, Zalutsky R. How common are the "common" neurologic disorders? Neurology. 2007;68:326-37.

2. Banerjee PN, Filippi D, Hauser WA. The descriptive epidemiology of epilepsy-a review. Epilepsy Res. 2009;85:31-45.

3. Cagnetti C, Lattanzi S, Foschi N, Provinciali L, Silvestrini M. Seizure course during pregnancy in catamenial epilepsy. Neurology. 2014;83:339-44.

4. Cockerell OC, Johnson AL, Sander JW, Hart YM, Shorvon SD. Remission of epilepsy: results from the national general practice study of epilepsy. Lancet. 1995;346:140-4.

5. Lattanzi S, Zaccara G, Giovannelli F, Grillo E, Nardone R, Silvestrini M, Trinka E, Brigo F. Antiepileptic monotherapy in newly 
diagnosed focal epilepsy. A network meta-analysis. Acta Neurol Scand. 2019;139:33-41.

6. Kwan P, Brodie MJ. Early identification of refractory epilepsy. N Engl J Med. 2000;342:314-9.

7. Lattanzi S, Cagnetti C, Foschi N, Provinciali L, Silvestrini M. Lacosamide monotherapy for partial onset seizures. Seizure. 2015;27:71-4

8. Kwok CS, Johnson EL, Krauss GL. Comparing safety and efficacy of "Third-Generation" antiepileptic drugs: long-term extension and post-marketing treatment. CNS Drugs. 2017;31:959-74.

9. Hutton B, Salanti G, Caldwell DM, et al. The PRISMA extension statement for reporting of systematic reviews incorporating network meta-analyses of health care interventions: checklist and explanations. Ann Intern Med. 2015;162:777-84.

10. Gazzola DM, Balcer LJ, French JA. Seizure-free outcome in randomised add-on trials of the new antiepileptic drugs. Epilepsia. 2007;48:1303-7.

11. Brivaracetam. Summary of product characteristic. https://www. ema.europa.eu/en/documents/product-information/briviact-eparproduct-information_en.pdf. Accessed July 2021.

12. Cenobamate. Summary of product characteristic. https://www. ema.europa.eu/en/documents/product-information/ontozry-eparproduct-information en.pdf. Accessed July 2021.

13. Eslicarbazepine acetate. Summary of product characteristic. https://www.ema.europa.eu/en/documents/product-information/ zebinix-epar-product-information_en.pdf. Accessed July 2021.

14. Lacosamide. Summary of product characteristic. https://www. ema.europa.eu/en/documents/product-information/vimpat-eparproduct-information_en.pdf. Accessed July 2021

15. Perampanel. Summary of product characteristic. https://www. ema.europa.eu/en/documents/product-information/fycompa-eparproduct-information_en.pdf. Accessed July 2021.

16. Cochrane Handbook for Systematic Reviews of Interventions Version 5.1.0 [updated March 2011]. Higgins JPT and Green S, editors. The Cochrane Collaboration, 2011. http://handbook-5-1. cochrane.org/. Accessed June 2021.

17. Nikolakopoulou A, Higgins JPT, Papakonstantinou T, Chaimani A, Del Giovane C, Egger M, Salanti G. CINeMA: an approach for assessing confidence in the results of a network meta-analysis. PLoS Med. 2020;17:e1003082.

18. Salanti G. Indirect and mixed-treatment comparison, network, or multiple-treatments meta-analysis: many names, many benefits, many concerns for the next generation evidence synthesis tool. Res Synth Methods. 2012;3:80-97.

19. Cipriani A, Higgins JP, Geddes JR, et al. Conceptual and technical challenges in network meta-analysis. Ann Intern Med. 2013;159:130-7.

20. Veroniki AA, Vasiliadis HS, Higgins JP, Salanti G. Evaluation of inconsistency in networks of interventions. Int J Epidemiol. 2013;42:332-45.

21. Biton V, Berkovic SF, Abou-Khalil B, Sperling MR, Johnson $\mathrm{ME}, \mathrm{Lu} \mathrm{S}$. Brivaracetam as adjunctive treatment for uncontrolled partial epilepsy in adults: a phase III randomised, double-blind, placebo-controlled trial. Epilepsia. 2014;55:57-66.

22. Ryvlin P, Werhahn KJ, Blaszczyk B, Johnson ME, Lu S. Adjunctive brivaracetam in adults with uncontrolled focal epilepsy: results from a double-blind, randomised, placebo-controlled trial. Epilepsia. 2014;55:47-56.

23. Klein P, Schiemann J, Sperling MR, Whitesides J, Liang W, Stalvey T, Brandt C, Kwan P. A randomised, double-blind, placebo-controlled, multicenter, parallel-group study to evaluate the efficacy and safety of adjunctive brivaracetam in adult patients with uncontrolled partial-onset seizures. Epilepsia. 2015;56:1890-8.

24. Krauss GL, Klein P, Brandt C, Lee SK, Milanov I, Milovanovic M, Steinhoff BJ, Kamin M. Safety and efficacy of adjunctive cenobamate (YKP3089) in patients with uncontrolled focal seizures: a multicentre, double-blind, randomised, placebo-controlled, dose-response trial. Lancet Neurol. 2020;19:38-48.

25. Elger C, Halász P, Maia J, Almeida L, Soares-da-Silva P, BIA2093-301 Investigators Study Group. Efficacy and safety of eslicarbazepine acetate as adjunctive treatment in adults with refractory partial-onset seizures: a randomised, double-blind, placebo-controlled, parallel-group phase III study. Epilepsia. 2009;50:454-63.

26. Gil-Nagel A, Lopes-Lima J, Almeida L, Maia J, Soares-da-Silva P, BIA-2093-303 Investigators Study Group. Efficacy and safety of 800 and $1200 \mathrm{mg}$ eslicarbazepine acetate as adjunctive treatment in adults with refractory partial-onset seizures. Acta Neurol Scand. 2009;120:281-7.

27. Ben-Menachem E, Gabbai AA, Hufnagel A, Maia J, Almeida L, Soares-da-Silva P. Eslicarbazepine acetate as adjunctive therapy in adult patients with partial epilepsy. Epilepsy Res. 2010;89:278-85.

28. Sperling MR, Abou-Khalil B, Harvey J, Rogin JB, Biraben A, Galimberti CA, Kowacs PA, Hong SB, Cheng H, Blum D, Nunes T, Soares-da-Silva P, 304 Study Team. Eslicarbazepine acetate as adjunctive therapy in patients with uncontrolled partial-onset seizures: results of a phase III, double-blind, randomised, placebocontrolled trial. Epilepsia. 2015;56:244-53.

29. Ben-Menachem E, Biton V, Jatuzis D, Abou-Khalil B, Doty P, Rudd GD. Efficacy and safety of oral lacosamide as adjunctive therapy in adults with partial-onset seizures. Epilepsia. 2007:48:1308-17.

30. Halász P, Kälviäinen R, Mazurkiewicz-Beldzińska M, Rosenow F, Doty P, Hebert D, Sullivan T, SP755 Study Group. Adjunctive lacosamide for partial-onset seizures: Efficacy and safety results from a randomised controlled trial. Epilepsia. 2009;50:443-53.

31. Chung S, Sperling MR, Biton V, Krauss G, Hebert D, Rudd GD, Doty P, SP754 Study Group. Lacosamide as adjunctive therapy for partial-onset seizures: a randomised controlled trial. Epilepsia. 2010;51:958-67.

32. Hong Z, Inoue Y, Liao W, Meng H, Wang X, Wang W, Zhou L, Zhang L, Du X, Tennigkeit F, EP0008 Study Group. Efficacy and safety of adjunctive lacosamide for the treatment of partial-onset seizures in Chinese and Japanese adults: A randomised, doubleblind, placebo-controlled study. Epilepsy Res. 2016;127:267-75.

33. French JA, Krauss GL, Biton V, Squillacote D, Yang H, Laurenza A, Kumar D, Rogawski MA. Adjunctive perampanel for refractory partial-onset seizures: randomised phase III study 304. Neurology. 2012;79:589-96.

34. French JA, Krauss GL, Steinhoff BJ, Squillacote D, Yang H, Kumar D, Laurenza A. Evaluation of adjunctive perampanel in patients with refractory partial-onset seizures: results of randomised global phase III study 305. Epilepsia. 2013;54:117-25.

35. Krauss GL, Serratosa JM, Villanueva V, Endziniene M, Hong Z, French J, Yang H, Squillacote D, Edwards HB, Zhu J, Laurenza A. Randomised phase III study 306: adjunctive perampanel for refractory partial-onset seizures. Neurology. 2012;78:1408-15.

36. Nishida T, Lee SK, Inoue Y, Saeki K, Ishikawa K, Kaneko S. Adjunctive perampanel in partial-onset seizures: Asia-Pacific, randomised phase III study. Acta Neurol Scand. 2018;137:392-9.

37. Chen Z, Brodie MJ, Liew D, Kwan P. Treatment outcomes in patients with newly diagnosed epilepsy treated with established and new antiepileptic drugs: a 30-year Longitudinal Cohort Study. JAMA Neurol. 2018;75:279-86.

38. Laxer KD, Trinka E, Hirsch LJ, Cendes F, Langfitt J, Delanty N, Resnick T, Benbadis SR. The consequences of refractory epilepsy and its treatment. Epilepsy Behav. 2014;37:59-70.

39. Roberti R, De Caro C, Iannone LF, Zaccara G, Lattanzi S, Russo E. Pharmacology of cenobamate: mechanism of action, 
pharmacokinetics, drug-drug interactions and tolerability. CNS Drugs. 2021;35:609-18.

40. Nakamura M, Cho JH, Shin H, Jang IS. Effects of cenobamate (YKP3089), a newly developed anti-epileptic drug, on voltagegated sodium channels in rat hippocampal CA3 neurons. Eur J Pharmacol. 2019;855:175-82.

41. Sharma R, Nakamura M, Neupane C, Jeon BH, Shin H, Melnick SM, Glenn KJ, Jang IS, Park JB. Positive allosteric modulation of GABA(A) receptors by a novel antiepileptic drug cenobamate. Eur J Pharmacol. 2020;879:173117.

42. Guignet M, Campbell A, White HS. Cenobamate (XCOPRI): Can preclinical and clinical evidence provide insight into its mechanism of action? Epilepsia. 2020;61:2329-39.

43. Lattanzi S, Cagnetti C, Foschi N, Provinciali L, Silvestrini M. Brivaracetam add-on for refractory focal epilepsy: a systematic review and meta-analysis. Neurology. 2016;86:1344-52.

44. Doty P, Hebert D, Mathy FX, Byrnes W, Zackheim J, Simontacchi K. Development of lacosamide for the treatment of partial-onset seizures. Ann N Y Acad Sci. 2013;1291:56-68.

45. Moseley BD, Chanteux H, Nicolas JM, Laloyaux C, Gidal B, Stockis A. A review of the drug-drug interactions of the antiepileptic drug brivaracetam. Epilepsy Res. 2020;163:106327.

46. Li-Na Z, Deng C, Hai-Jiao W, Da X, Ge T, Ling L. Indirect comparison of third-generation antiepileptic drugs as adjunctive treatment for uncontrolled focal epilepsy. Epilepsy Res. 2018;139:60-72.

47. Brigo F, Bragazzi NL, Nardone R, Trinka E. Efficacy and tolerability of brivaracetam compared to lacosamide, eslicarbazepine acetate, and perampanel as adjunctive treatments in uncontrolled focal epilepsy: results of an indirect comparison meta-analysis of RCTs. Seizure. 2016;42:29-37.

48. Lattanzi S, Canafoglia L, Canevini MP, Casciato S, Chiesa V, Dainese F, De Maria G, Didato G, Falcicchio G, Fanella M, Ferlazzo E, Fisco G, Gangitano M, Giallonardo AT, Giorgi FS, La Neve A, Mecarelli O, Montalenti E, Piazza F, Pulitano P, Quarato PP, Ranzato F, Rosati E, Tassi L, Di Bonaventura C. Adjunctive brivaracetam in focal epilepsy: real-world evidence from the BRIVAracetam add-on First Italian netwoRk STudy (BRIVAFIRST). CNS Drugs. 2021. https://doi.org/10.1007/s40263-021-00856-3 (Online ahead of print).

49. Steinhoff BJ, Klein P, Klitgaard H, Laloyaux C, Moseley BD, Ricchetti-Masterson K, Rosenow F, Sirven JI, Smith B, Stern JM, Toledo M, Zipfel PA, Villanueva V. Behavioral adverse events with brivaracetam, levetiracetam, perampanel, and topiramate: a systematic review. Epilepsy Behav. 2021;118:107939.

50. Hebeisen S, Pires N, Loureiro AI, Bonifácio MJ, Palma N, Whyment A, Spanswick D, Soares-da-Silva P. Eslicarbazepine and the enhancement of slow inactivation of voltage-gated sodium channels: a comparison with carbamazepine, oxcarbazepine and lacosamide. Neuropharmacology. 2015;89:122-35.

51. Chung SS, French JA, Kowalski J, Krauss GL, Lee SK, Maciejowski M, Rosenfeld WE, Sperling MR, Mizne S, Kamin M. Randomised phase 2 study of adjunctive cenobamate in patients with uncontrolled focal seizures. Neurology. 2020;94:e2311-22.

52. Trinka E, Tsong W, Toupin S, Patten A, Wilson $\mathrm{K}$, Isojarvi J, James D. A systematic review and indirect treatment comparison of perampanel versus brivaracetam as adjunctive therapy in patients with focal-onset seizures with or without secondary generalization. Epilepsy Res. 2020;166:106403.

53. Guyatt G, Jaeschke R, Heddle N, Cook D, Shannon H, Walter S. Basic statistics for clinicians: 1. Hypothesis testing. CMAJ. 1995;152:27-32.

54. Song F, Altman DG, Glenny AM, Deeks JJ. Validity of indirect comparison for estimating efficacy of competing interventions: empirical evidence from published meta-analyses. BMJ. 2003;326:472-5.

55. European Medicines Agency. Committee for medicinal products for human use. Guideline on clinical investigation of medicinal products in the treatment of epileptic disorders. https://www.ema. europa.eu/en/documents/scientific-guideline/guideline-clinicalinvestigation-medicinal-products-treatment-epileptic-disordersrevision-2_en.pdf. Accessed Oct 2021.

56. Verrotti A, Lattanzi S, Brigo F, Zaccara G. Pharmacodynamic interactions of antiepileptic drugs: From bench to clinical practice. Epilepsy Behav. 2020;104(Pt A):6939.

57. Fisher RS, Blum DE, DiVentura B, Vannest J, Hixson JD, Moss R, Herman ST, Fureman BE, French JA. Seizure diaries for clinical research and practice: limitations and future prospects. Epilepsy Behav. 2012;24:304-10.

58. Lattanzi S, De Maria G, Rosati E, Didato G, Chiesa V, Ranzato F, Canafoglia L, Cesnik E, Anzellotti F, Meletti S, Pauletto G, Nilo A, Bartolini E, Marino D, Tartara E, Luisi C, Bonanni P, Marrelli A, Stokelj D, Dainese F. Brivaracetam as add-on treatment in focal epilepsy: a real-world time-based analysis. Epilepsia. 2021;62:e1-6.

59. Brigo F, Del Giovane C, Nardone R, Trinka E, Lattanzi S. Intravenous antiepileptic drugs in adults with benzodiazepine-resistant convulsive status epilepticus: a systematic review and network meta-analysis. Epilepsy Behav. 2019;101(Pt B):106466.

60. Lattanzi S, Trinka E, Del Giovane C, Nardone R, Silvestrini M, Brigo F. Antiepileptic drug monotherapy for epilepsy in the elderly: a systematic review and network meta-analysis. Epilepsia. 2019;60:2245-54. 


\section{Authors and Affiliations}

\section{Simona Lattanzi ${ }^{1}\left({ }^{10} \cdot\right.$ Eugen Trinka $^{2,3,4} \cdot$ Gaetano Zaccara $^{5} \cdot$ Pasquale Striano $^{6} \cdot$ Emilio Russo $^{7} \cdot$ Cinzia Del Giovane ${ }^{8}$. Mauro Silvestrini ${ }^{1} \cdot$ Francesco Brigo $^{9,10}$}

1 Department of Experimental and Clinical Medicine, Neurological Clinic, Marche Polytechnic University, Via Conca 71, 60020 Ancona, Italy

2 Department of Neurology, Christian Doppler Klinik, Paracelsus Medical University, Salzburg, Austria

3 Center for Cognitive Neuroscience, Salzburg, Austria

4 Public Health, Health Services Research and HTA, University for Health Sciences, Medical Informatics and Technology, Hall in Tirol, Austria

5 Health Agency of Tuscany, Florence, Italy

6 Pediatric Neurology and Muscular Diseases Unit, Department of Neurosciences, Rehabilitation,
Ophthalmology, Genetics, Maternal and Child Health, "G. Gaslini”" Institute, University of Genova, Genova, Italy

7 Science of Health Department, University Magna Grecia of Catanzaro, Catanzaro, Italy

8 Institute of Primary Health Care (BIHAM), University of Bern, Bern, Switzerland

9 Department of Neuroscience, Biomedicine and Movement Science, University of Verona, Verona, Italy

10 Division of Neurology, "Franz Tappeiner" Hospital, Merano, BZ, Italy 